\title{
Gapped boundary theory of the twisted gauge theory model of three-dimensional topological orders
}

\author{
Hongyu Wang, ${ }^{a, b}$ Yingcheng $\mathbf{L i},^{a, b}$ Yuting $\mathbf{H u}^{c}$ and Yidun Wan ${ }^{a, b, c, d, e, 1}$ \\ ${ }^{a}$ State Key Laboratory of Surface Physics, Fudan University, \\ Shanghai 200433, China \\ ${ }^{b}$ Department of Physics and Center for Field Theory and Particle Physics, Fudan University, \\ Shanghai 200433, China \\ ${ }^{c}$ Department of Physics and Institute for Quantum Science and Engineering, \\ Southern University of Science and Technology, \\ Shenzhen 518055, China \\ ${ }^{d}$ Institute for Nanoelectronic devices and Quantum computing, Fudan University, \\ Shanghai 200433, China \\ ${ }^{e}$ Collaborative Innovation Center of Advanced Microstructures, Nanjing University, \\ Nanjing 210093, China \\ E-mail: 17110190004@fudan.edu.cn, 17210190004@fudan.edu.cn, \\ yuting.phys@gmail.com, ydwan@fudan.edu.cn
}

ABSTRACT: We extend the twisted gauge theory model of topological orders in three spatial dimensions to the case where the three spaces have two dimensional boundaries. We achieve this by systematically constructing the boundary Hamiltonians that are compatible with the bulk Hamiltonian. Given the bulk Hamiltonian defined by a gauge group $G$ and a fourcocycle $\omega$ in the fourth cohomology group of $G$ over U(1), we construct a gapped boundary Hamiltonian using $\{K, \alpha\}$, with a subgroup $K \subseteq G$ and a 3-cochain $\alpha$ of $K$ over U(1), which satisfies the generalized Frobenius condition. The Hamiltonian is invariant under the topological renormalization group flow (via Pachner moves). Each solution $\{K, \alpha\}$ to the generalized Frobenius condition specifies a gapped boundary condition. We derive a closed-form formula of the ground state degeneracy of the model on a three-cylinder, which can be naturally generalized to three-spaces with more boundaries. We also derive the explicit ground-state wavefunction of the model on a three-ball. The ground state degeneracy and ground-state wavefunction are both presented solely in terms of the input data of the model, namely, $\{G, \omega, K, \alpha\}$.

Keywords: Anyons, Lattice Quantum Field Theory, Topological Field Theories, Topological States of Matter

ARXIV EPRINT: 1807.11083

\footnotetext{
${ }^{1}$ Corresponding author.
} 


\section{Contents}

1 Introduction 1

2 Review of the TGT model 3

3 The TGT model with boundaries $\quad 8$

4 One-to-one correspondence between 3-cochain and 3-cocycle 12

5 Ground-state wavefunctions on a 3-ball 12

6 GSD on a 3-cylinder $\quad 15$

7 Examples: $G=\mathbb{Z}_{2} \times \mathbb{Z}_{2} \times \mathbb{Z}_{2}$

A A brief introduction to cohomology groups $H^{n}[G, \mathrm{U}(1)] \quad 17$

$\begin{array}{ll}\text { B Some properties about } A_{v}^{K} \text { operators } & 18\end{array}$

C The derivation of GSD on a 3-cylinder 19

\section{Introduction}

Systems bearing intrinsic topological orders in two [1-16] and three [15, 17-29] spatial dimensions have received substantial attention recently because they not only have greatly expanded and deepened our understanding of phases of matter but also have practical applications, in particular certain 2-dimensional topologically ordered systems can support topological quantum computation [5, 30]. Celebrated candidates of two-dimensional topological phases include chiral spin liquids $[2,31], \mathbb{Z}_{2}$ spin liquids [32-34], Abelian quantum Hall states [35-37], and non-Abelian fractional quantum Hall states [38-42].

Although three is our physical spatial dimension, our understanding of threedimensional topological orders is far behind that of the two-dimensional ones. Moreover, candidates of three-dimensional topological orders are still missing. Recent works also show that three-dimensional topological orders are related to quantum gravity $[43,44]$. It is therefore demanding and interesting to study theories and models of three-dimensional topological orders. Symmetry considerations have led to the twisted gauge theory (TGT) model that describes and classifies a large class of three-dimensional topological orders $[19,21]$. The TGT model is an exactly solvable lattice Hamiltonian extension of the $(3+1)$-dimensional Dijkgraaf-Witten topological gauge theory with general finite gauge 
groups on a lattice. The TGT model is specified by a Hamiltonian $H^{G, \omega}$ defined on a triangulation of a 3-manifold, where $G$ is a finite gauge group, and the usual gauge transformation is twisted by a $\mathrm{U}(1) 4$-cocycle $\omega \in H^{4}[G, \mathrm{U}(1)]$, the fourth cohomology group of $G$. This model however cannot handle the situation where the 3-manifolds have boundaries, whereas realistic materials - in particular in three dimensions - mostly do have boundaries.

When boundaries are present, the Hamiltonian of the model should contain boundary terms too. For the model to be able to describe topological orders with gapped boundaries, ${ }^{1}$ we only consider the boundary terms that can render the boundaries gapped. Such boundary terms may affect the spectrum of the model in two aspects. First, the topologically protected ground state degeneracy (GSD) of a topological order may be modified due to its gapped boundary conditions. Second, different gapped boundary conditions correspond to different sets of bulk excitations condensing at the boundaries [45-50]. These two aspects had been an open problem of topological orders in two and three dimensions. Until only recently these two problems in two-dimensions have been solved for Abelian topological orders [51] and for general, non-Abelian topological orders $[49,52,53]$ but are still open for three-dimensions.

More broadly, on the one hand, a dynamical theory incapable of handling boundary conditions is not a complete dynamical theory. Therefore, the TGT model is not yet a complete dynamical model on open three-manifolds. On the other hand, the bulk and boundary relation in light of anyon condensation (in any dimensions [50]) offers a new perspective of holographic principle, which may shed new light on the AdS/CFT correspondence. ${ }^{2}$

Recently, there have been a few works $[45,50,54-58]$ dealing with the boundary theories of topological orders in two and higher dimensions. They either take an algebraic approach $[50,54,55]$, or study a particular interesting example [57], or focus on symmetry (protected) enriched topological orders [56, 58]. Nevertheless, we shall generalize the systematic approach of exactly solvable Hamiltonian developed by two of us in refs. [53, 59, 60], where the twisted quantum double model and the string-net model of two-dimensional topological orders are extended to include boundaries. In this paper, we extend the three-dimensional TGT model to include boundaries. We shall restrict the boundary degrees of freedom in the extended TGT model to take value in $K \subseteq G$. We then add to the original TGT Hamiltonian compatible boundary terms that depend on $K$ and a 3-cochain $\alpha \in C^{3}[K, \mathrm{U}(1)]$. Studying this extended TGT model leads to the following main results.

We extend the TGT bulk Hamiltonian by a local boundary Hamiltonian with boundary degrees of freedom being in a subgroup (not necessarily a proper one) of the gauge group in the bulk. The local operators in boundary Hamiltonian are constructed in terms of a 3-cochain of the boundary subgroup. The boundary local Hamiltonian needs to be exactly solvable to make sure that the model describes gapped boundaries. The boundary Hamiltonian should also be compatible with the bulk Hamiltonian, such that the ground states are invariant under topology-preserving mutation of triangulation both in the bulk

\footnotetext{
${ }^{1}$ So as the topological orders to have well defined ground state degeneracy.

${ }^{2}$ Via private communication with Ling-Yan Hung.
} 


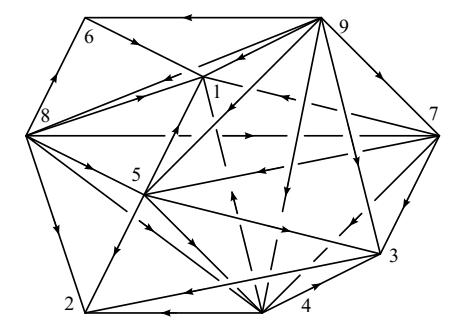

Figure 1. A portion of a graph that represent the basis vectors in the Hilbert space. Each edge carries an arrow and is assigned a group element denoted by $[a b]$ with $a<b$.

and on the boundary. These requirements will result in what we call the generalized Frobenius condition (as a generalization of the two-dimensional version [53]) that dictates the gapped boundary conditions.

Our extended Hamiltonian has the advantage that enables us to write down an explicit ground-state wavefunction of our model on a 3-ball and a closed-form formula for the GSD on a 3-cylinder, both are cast in terms of the input data of the model only, namely, $\{G, \omega, K, \alpha\}$. We show a couple of examples.

The paper is organized as follows. Section 2 briefly reviews the TGT model. Sections 3 and 4 systematically construct the boundary Hamiltonian. Section 5 derives the explicit ground-state wavefunction of our model on a 3-ball. Section 6 deduces the closed-form formula of the ground state degeneracy (GSD) of our model on a 3-cylinder. The appendices include a basic introduction to group cohomology and certain details too much to appear in the main text.

\section{Review of the TGT model}

Here we briefly review the TGT model of topological orders on closed 3-manifold. The TGT model is defined by a low-energy effective Hamiltonian $H_{G, \omega}$ on a triangulation $\Gamma$ (see figure 1) of a closed, orientable, 3-manifold, e.g., a 3-sphere and a 3-torus. Each edge $a b$ from vertex $a$ to vertex $b$ in $\Gamma$ is graced with a group element $[a b] \in G$, rendering the Hilbert space of the model consists of all possible configurations of the group elements on the edges of $\Gamma$. Namely,

$$
\mathcal{H}_{\Gamma, G}=\{[i j] \in G \mid i, j \in V(\Gamma)\},
$$

where $V(\Gamma)$ is the set of vertices of $\Gamma$. The states are orthogonal obviously. The group elements on the edges can be considered as the discretized gauge field of the underlying Dijkgraaf-Witten topological gauge theory. The graph is oriented with an arbitrary ordering of the vertices, ${ }^{3}$ such that each edge is arrowed from its larger vertex to the smaller and that $[a b]=\overline{[b a]}$, where the overline denotes the group inverse. A vertex ordering is called an enumeration [11] and does not affect the physics as long as the relative order of the vertices persists when the graph mutates. A graph $\Gamma$ mutates via the 3 -dimensional

\footnotetext{
${ }^{3}$ The corresponding triangulation is said to have a branching structure.
} 
Pachner moves [61, 62], seen in eq. (2.2).

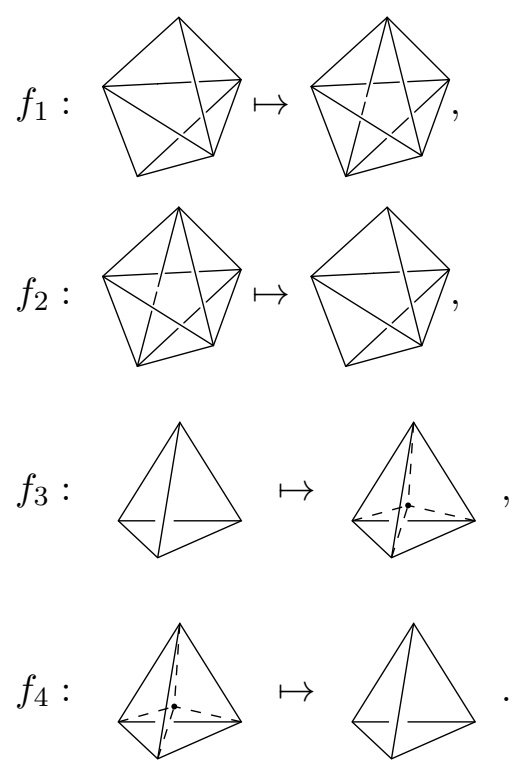

The mutation of $\Gamma$ turns $\Gamma$ into a different graph $\Gamma^{\prime}$ and hence alters the total Hilbert space of the model. Nevertheless, as shown in ref. [11], the topological properties of the topological order described by the model $H_{G, \omega}$ do not change because mutations cannot alter the topology of the surface. For simplicity, we neglect drawing the group elements on the edges but keep only the vertex labels. We may also refer to $a b c$ as a 2 -simplex, $a b c d$ as a 3 -simplex, and $[a b c d]$ as a 3 -cocycle on the tetrahedron. On any part of $\Gamma$ that resembles figure 2(a), one can define a normalized 4-cocycle $\left[v_{1} v_{2} v_{3} v_{4} v_{5}\right] \in H^{4}[G, \mathrm{U}(1)]$. The four group variables in the 4 -cocycle from left to right are respectively $\left[v_{1} v_{2}\right],\left[v_{2} v_{3}\right],\left[v_{3} v_{4}\right]$, and $\left[v_{4} v_{5}\right]$, which are along the path from the least vertex $v_{1}$ to the greatest vertex $v_{5}$ passing $v_{2}$ and $v_{3}$ in order. Appendix A reviews necessary rudiments of group cohomology. Here one should keep in mind that a 4-cocycle is an equivalence class of $\mathrm{U}(1)$-valued functions on $G^{4}=G \times G \times G \times G$. A normalized 4-cocycle is a particular representative that satisfies the normalization condition

$$
\left[v_{1} v_{2} v_{3} v_{4} v_{5}\right]=1 \text { if }\left[v_{i} v_{i+1}\right]=1 \in G \text { for any } i
$$

and the 4-cocyle condition

$$
\Pi_{i=1}^{5}(-1)^{i+1}\left[v_{1} \ldots \hat{v}_{i} \ldots v_{5}\right]=1
$$

where $\hat{v}_{i}$ means removing the point $v_{i}$. We take this notation in order to figure out easily the triangles required to be flat (defined in eq. (2.7)) when we use Pachner moves. To make sure all the elements multiplication is allowed, one need to check if all the triangles $v_{i-1} v_{i} v_{i+1}(i=2, \ldots, 4)$ are flat eq. $(2.7)$.

Shown in ref. [11], each 4-cocycle defines a 3 -dimensional topological order and the choice of the normalized 4-cocycle as the representative is merely a convenience that does not affect the physics. A graph like figure 2(a) has an associated 4-cocyle whose orientation 


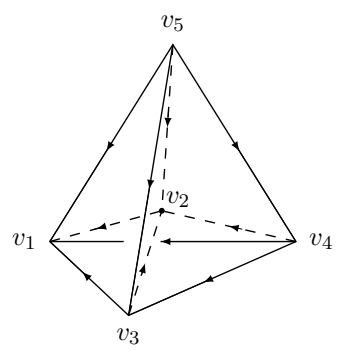

(a)

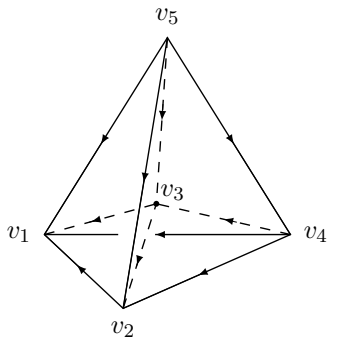

(b)

Figure 2. (a) The defining graph of the 4-cocycle $\left[v_{1} v_{2} v_{3} v_{4} v_{5}\right]$. (b) Of $\left[v_{1} v_{2} v_{3} v_{4} v_{5}\right]^{-1}$.

determined as follows. One first reads off a list of the vertices from any of the four tetrahedra of the defining graph of the 4-cocycle, e.g., the $v_{2} v_{3} v_{4} v_{5}$ from figure 2 (a) and $v_{3} v_{2} v_{4} v_{5}$ from figure 2(b). One then appends the remaining vertex to the beginning of the list, e.g., the $v_{1} v_{2} v_{3} v_{4} v_{5}$ from figure $2(\mathrm{a})$ and $v_{1} v_{3} v_{2} v_{4} v_{5}$ from figure 2(b). If the list can be reordered ascendingly by even permutations, such as $v_{1} v_{2} v_{3} v_{4} v_{5}$ from figure $2(\mathrm{a})$, one has the 4-cocycle $\left[v_{1} v_{2} v_{3} v_{4} v_{5}\right]$, otherwise $\left[v_{1} v_{2} v_{3} v_{4} v_{5}\right]^{-1}$ as by the $v_{1} v_{3} v_{2} v_{4} v_{5}$ from figure $2(\mathrm{~b})$.

The Hamiltonian of our model reads

$$
H_{G, \omega}=-\sum_{v} A_{v}-\sum_{f} B_{f}
$$

where $B_{f}$ are the face operators defined on the triangular faces $f$, and $A_{v}$ are the vertex operators defined at the vertices $v$. The operator $B_{f}$ acts on the face $f$ in a basis state vector as

$$
B_{f}\left|\bigwedge_{v_{1}}^{v_{3}}\right\rangle=\delta_{\left[v_{1} v_{2}\right] \cdot\left[v_{2} v_{3}\right] \cdot\left[v_{3} v_{1}\right]}\left|\bigwedge_{v_{1}}^{v_{3}}\right\rangle \text {. }
$$

The discrete delta function $\delta_{\left[v_{1} v_{2}\right] \cdot\left[v_{2} v_{3}\right] \cdot\left[v_{3} v_{1}\right]}$ is unity if $\left[v_{1} v_{2}\right] \cdot\left[v_{2} v_{3}\right] \cdot\left[v_{3} v_{1}\right]=1$, where 1 is the identity element in $G$, and 0 otherwise. Note again that here, the ordering of $v_{1}, v_{2}$, and $v_{3}$ is irrelevant because of the identities $\delta_{\left[v_{1} v_{2}\right] \cdot\left[v_{2} v_{3}\right] \cdot\left[v_{3} v_{1}\right]}=\delta_{\left[v_{3} v_{1}\right] \cdot\left[v_{1} v_{2}\right] \cdot\left[v_{2} v_{3}\right]}$ and $\delta_{\left[v_{1} v_{2}\right] \cdot\left[v_{2} v_{3}\right] \cdot\left[v_{3} v_{1}\right]}=\delta_{\left\{\overline{\left.\left[v_{1} v_{2}\right] \cdot\left[v_{2} v_{3}\right] \cdot\left[v_{3} v_{1}\right]\right\}}\right.}=\delta_{\left.\overline{\left[v_{3} v_{1}\right]} \cdot \overline{\left[v_{2} v_{3}\right]}\right] \overline{\left[v_{1} v_{2}\right]}}=\delta_{\left[v_{1} v_{3}\right] \cdot\left[v_{3} v_{2}\right] \cdot\left[v_{2} v_{1}\right]}$. In other words, if $B_{f}=1$ on a face $f$, the three group elements on the three edges of $f$ are related by a chain rule:

$$
\left[v_{1} v_{3}\right]=\left[v_{1} v_{2}\right] \cdot\left[v_{2} v_{3}\right]
$$

for any enumeration $v_{1}, v_{2}, v_{3}$ of the three vertices of the face $f$. The chain rule (2.7) is physically known as the flatness condition in the sense that the gauge connection along the edges of a triangular face is flat. The operator $A_{v}$ acting on a vertex $v$ is an average

$$
A_{v}=\frac{1}{|G|} \sum_{\left[v v^{\prime}\right]=g \in G} A_{v}^{g}
$$

over the operators $A_{v}^{g}$ specified by a group element $g \in G$ acting on the same vertex. The action of $A_{v}^{g}$ replaces $v$ by a new enumeration $v^{\prime}$ that is less than $v$ but greater than all the 
vertices that are less than $v$ in the original set of enumerations before the action, such that $\left[v^{\prime} v\right]=g$. In a dynamical language, $v^{\prime}$ may be thought as on the next "time" slice, and there is an edge $\left[v^{\prime} v\right] \in G$ in the (3+1)-dimensional "spacetime" picture. We illustrate such an action in the example below, which suffices to show the general definition on arbitrary vertex.

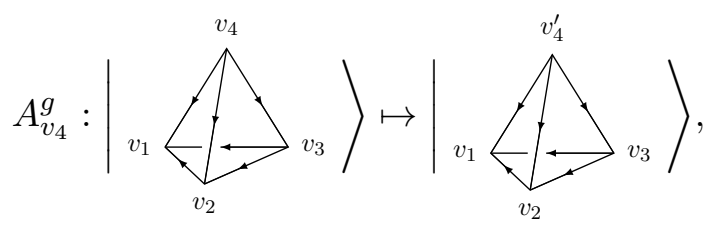

where on the r.h.s., the new enumerations are in the order $v_{1}<v_{2}<v_{3}<v_{4}^{\prime}<v_{4}$, satisfying the following flatness conditions.

$$
\begin{aligned}
& {\left[v_{1} v_{4}^{\prime}\right]=\left[v_{1} v_{4}\right] \cdot\left[v_{4} v_{4}^{\prime}\right],} \\
& {\left[v_{2} v_{4}^{\prime}\right]=\left[v_{2} v_{4}\right] \cdot\left[v_{4} v_{4}^{\prime}\right],} \\
& {\left[v_{3} v_{4}^{\prime}\right]=\left[v_{3} v_{4}\right] \cdot\left[v_{4} v_{4}^{\prime}\right] .}
\end{aligned}
$$

Now imagine to put together the two tetrahedra before and after the action of $A_{v_{4}}^{g}$ as two spatial slices connected by the 'temporal' edge $v_{4}^{\prime} v_{4}$, which is not shown, we obtain a 4simplex. This is a picture of time evolution, which motivates us to attribute the amplitude of $A_{v_{4}}^{g}$ to an evaluation of the 4 -simplex. This amplitude is naturally given by the 4 -cocycle associated with the 4-simplex (recall our earlier discussion). That is,

$$
\begin{aligned}
& \left\langle v_{1} \bigcap_{v_{3}}\left|A_{v_{4}}^{g}\right| v_{1}\left\langle v_{v_{2}}^{v_{4}^{\prime}}\right\rangle\right. \\
& =\delta_{\left[v_{4}^{\prime} v_{4}\right], g}\left\langle v_{v_{1}}\right. \\
& =\delta_{\left[v_{4}^{\prime} v_{4}\right], g}\left[v_{1} v_{2} v_{3} v_{4}^{\prime} v_{4}\right]^{\epsilon\left(v_{1} v_{2} v_{3} v_{4}^{\prime} v_{4}\right)} \\
& =\delta_{\left[v_{4}^{\prime} v_{4}\right], g}\left[v_{1} v_{2} v_{3} v_{4}^{\prime} v_{4}\right]^{-1}
\end{aligned}
$$

The 4-cocycles appearing on the r.h.s. of eq. (2.11) are understood from figure 3. This figure illustrates the time evolution of the graph due to the action of $A_{v_{4}}^{g}$. To define $\epsilon\left(v_{1} v_{2} v_{3} v_{4}^{\prime} v_{4}\right)$, we first need to define the orientation of tetrahedron, $\epsilon\left(v_{1} v_{2} v_{3} v_{4}\right)$. The convention is as follows.

Convention 1. One can grab the triangle of $v_{1} v_{2} v_{3} v_{4}$ that does not contain the largest vertex, i.e., the triangle $v_{1} v_{2} v_{3}$, along its boundary, such that the three vertices are in ascending order, while the thumb points to the vertex $v_{4}$. If one must use one's right hand to achieve this, $v_{1} v_{2} v_{3} v_{4}$ 's orientation is + , or $\epsilon\left(v_{1} v_{2} v_{3} v_{4}\right)=1$, otherwise - , or $\epsilon\left(v_{1} v_{2} v_{3} v_{4}\right)=-1$. 


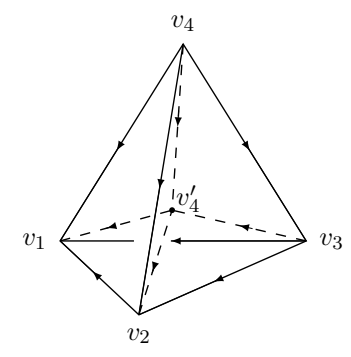

Figure 3. The topology of the action of $A_{v_{4}}^{g}$.

We then have the following definition of $\epsilon\left(v_{1} v_{2} v_{3} v_{4}^{\prime} v_{4}\right)$,

Convention 2. Since the new vertex $v_{4}^{\prime}$ is set to be slightly off the $3 d$ space of the tetrahedron $v_{1} v_{2} v_{3} v_{4}$, and since every newly created vertex bears a label slightly less than that of the original vertex acted on by the vertex operator, one can always choose the convention such that $\epsilon\left(v_{1} v_{2} v_{3} v_{4}^{\prime} v_{4}\right)=\epsilon\left(v_{1} v_{2} v_{3} v_{4}\right) \operatorname{sgn}\left(v_{4}^{\prime}, v_{1}, v_{2}, v_{3}, v_{4}\right)$. And $\operatorname{sgn}\left(v_{4}^{\prime}, v_{1}, v_{2}, v_{3}, v_{4}\right)$ is the sign of the permutation that takes the list of vertices in the argument to purely ascending as $\left(v_{1}, v_{2}, v_{3}, v_{4}^{\prime}, v_{4}, v_{5}\right)$, which embraces the 4-simplex $v_{1} v_{2} v_{3} v_{4}^{\prime} v_{4}$.

The vertex operator in eq. (2.11) can naturally extend its definition from a trivalent vertex to a vertex $v$ of any valence. The number of 4 -cocyles in the phase factor brought by the action of $A_{v}^{g}$ on $v$ equals $v$ 's valence of. It is clear that $A_{v}^{g=1} \equiv \mathbb{I}$ by the normalization of 4-cocycles. The Hamiltonian (2.5) is exactly solvable when all the triangles are flat, namely, in the Hilbert subspace $\mathcal{H}^{B_{f}=1}$. The ground states are the common +1 eigenvectors of these projectors. On a 3-torus, a ground-state basis state can be labeled by $|A, B, \mu\rangle$, where $A$ is a conjugacy class of $G, B$ is a conjugacy class in the centralizer subgroup $Z^{A}$, and $\mu$ an irreducible representation of the centralizer of both $A$ in $G$ and $B$ in $Z^{A}$. When both $A$ and $B$ are nontrivial, the excitations are loops instead of points because $A$ and $B$ are the bases of fluxes in two directions. The ground states $|A, B, \mu\rangle$ would cease to form a quantum-

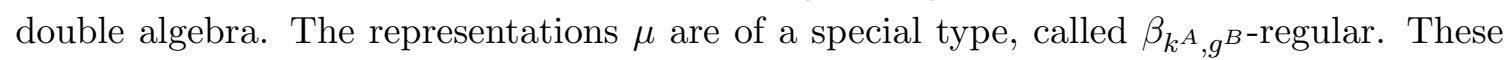
$\beta_{k^{A}, g^{B}}$ are doubly-twisted 2-cocycles derived from $\omega$ via the slant product (see appendix 1). Interestingly, the topological orders described by the TGT model are not classified by the 4-cocycles $w \in H^{4}[G, \mathrm{U}(1)]$ given $G$ but instead classified by the doubly-twisted 2-cocycles $\beta_{k^{A}, g^{B}}$ derived from $\omega$. [11] On a 3-torus, the GSD of the model $H_{G, \omega}$ is

$$
\mathrm{GSD}=\sum_{A=1}^{r(G)} \sum_{B=1}^{r\left(Z^{A}\right)} \#\left(\beta_{k^{A}, g^{B}}-\text { regular irreps of } Z^{A, B}\right),
$$

where the two sums run over all the conjugacy classes of $G$ and $Z^{A}$, \# denotes "the number of", and $Z^{A, B} \subset G$ is the centralizer of both $A$ and $B$. Each $\beta_{a, b}$ specifies a class of projective representations of $Z_{a, b}$ dubbed $\beta_{a, b}$-representations, which are defined by

$$
\tilde{\rho}^{a, b}(x) \tilde{\rho}^{a, b}(y)=\beta_{a, b}(x, y) \tilde{\rho}^{a, b}(x y) .
$$




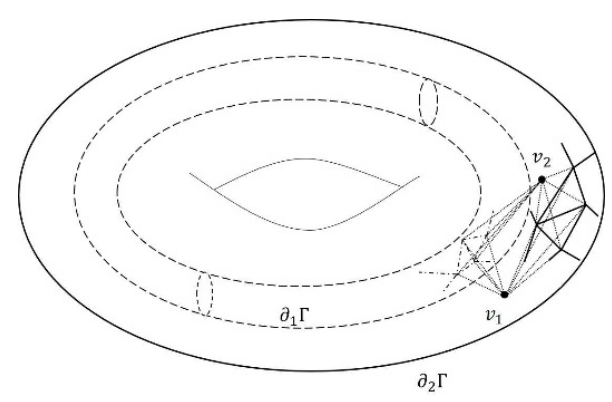

Figure 4. A 3-cylinder, i.e., a solid torus with a solid torus removed from interior. This is an example of a 3-manifold with two boundaries, the inner boundary (dashed line) $\partial_{1} \Gamma$ and the outter boundary (solid line) $\partial_{2} \Gamma$. A portion of the triangulation $\Gamma$ of this 3 -cylinder is shown, where $v_{1}$ and $v_{2}$ are two vertices in the bulk.

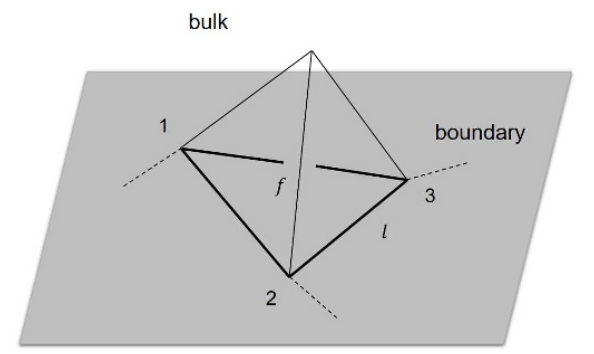

Figure 5. The boundary is the grey plane, above which is the bulk. A bulk tetrahedron and a boundary face $f=123$ is explicitly shown. The dashed lines represent the rest of the graph that is now drawn.

An element $c \in Z_{a, b}$ is called $\beta_{a, b}$-regular if $\beta_{a, b}(c, d)=\beta_{a, b}(d, c), \forall d \in Z_{a, b, c} \subseteq Z_{a, b}$. The $\beta_{a, b}$-regular irreducible representations are no other than the irreducible $\beta_{a, b}$-representations of the elements that are $\beta_{a, b}$-regular.

Unlike the $2+1$-dimensional TQD model on a torus, the ground states and quasiexcitations don't remain in one-to-one correspondence for $3+1$-dimensional TGT models on a 3 -torus.

\section{The TGT model with boundaries}

We now try to extend the TGT model reviewed above to one that is defined on a 3 -dimensional space with boundaries (see figure 4 for an example). We do not distinguish multiple boundaries from a single boundary that has multiple components.

Let us consider a portion of boundary graph shown in figure 5 with three boundary vertices $1,2,3$. There is a boundary plaquette $f=123$ with all the three edges on the boundary.

We first need to specify the degrees of freedom living on the boundaries. Since we require the boundaries to be gapped for the topological GSD to be well defined, the boundary degrees of freedom would naturally arise from those in the bulk. To be precise, consider a single boundary $\partial \Gamma$ of a graph $\Gamma$, if the bulk degrees of freedom take value in a finite group 
$G$, the degrees of freedom on $\partial \Gamma$ in the ground state space would take value in a subgroup $K \subseteq G$. This will become clear with a projection operator $C$ in the Hamiltonian as defined below. As in the $(2+1)$-dimensional cases, $K$ is allowed to be the trivial subgroup $\{1\}$ and $G$ itself too. If $\Gamma$ has $M$ boundaries $\partial_{1} \Gamma, \partial_{2} \Gamma, \ldots, \partial_{M} \Gamma$, the subgroups $K_{1}, K_{2}, \ldots, K_{n} \subseteq G$ are not necessarily the same or different. Having set up the boundary degrees of freedom, we can now propose the Hamiltonian of the TGT model with multiple boundaries:

$$
H_{G, \omega}^{K, \alpha}=H_{G, \omega}-\sum_{i=1}^{M}\left(\sum_{v \in \partial_{i} \Gamma} A_{v}^{K_{i}}-\sum_{f \in \partial_{i} \Gamma} B_{f}^{K_{i}}-\sum_{l \in \partial_{i} \Gamma} C_{l}^{K_{i}}\right),
$$

where $H_{G, \omega}$ is the bulk Hamiltonian (2.5), and the rest are the boundary terms to be explained in order.

An operator $B_{f}^{K}$ acts on a boundary plaquette $f$ and is defined as in the example below.

$$
B_{123}^{K}|\overbrace{i}>_{5^{-}}\rangle=\delta_{[12] \cdot[23] \cdot[31]}|\overbrace{5^{\prime}}\rangle
$$

This $\delta$ function is 1 if [12] $\cdot[23] \cdot[31]=1 \in K$ and 0 otherwise. An operator $C_{l}^{K}$ projects the degrees of freedom on the boundary edge $l$ into the subgroup $K$, namely,

$$
C_{l}^{K}=\sum_{k \in K} C_{l}^{k}
$$

Clearly, $\left[B_{f}^{K}, B_{f^{\prime}}^{K}\right]=0$ and $\left[C_{l}^{K}, C_{l}^{K}\right]=0$. Also all the boundary plaquette operators and boundary edge operators are projectors with the definition above.

Similar to a bulk vertex operator, a boundary vertex operator $A_{v}^{K}$ imposes a gauge transformation on the group elements on the edges incident at the boundary vertex $v$ :

$$
\begin{gathered}
A_{v}^{K}=\sum_{k \in K} A_{v}^{k}, \\
\left.\left.A_{v}^{k}\right\rangle_{i}\right\rangle=\mathcal{A}([0 v],[v 2],[23], k)
\end{gathered}
$$

where the ordering $0<v<2<3$ is assumed, and 0 is a bulk vertex. We relabeled the vertex $v$ by $v^{\prime}$, so that the new group elements on the edges after acting $A_{v}^{k}$ can be expressed by $\left[0 v^{\prime}\right],\left[v^{\prime} 2\right],\left[v^{\prime} 3\right]$ etc., which are defined by the following chain rules:

$$
\left[0 v^{\prime}\right] \cdot\left[v^{\prime} v\right]=[0 v],\left[v^{\prime} v\right] \cdot[v 2]=\left[v^{\prime} 2\right],\left[v^{\prime} v\right] \cdot[v 3]=\left[v^{\prime} 3\right]
$$


where we set $\left[v^{\prime} v\right]=k$. In eq. $(3.4), \mathcal{A}([0 v],[v 2],[23], k)$ is the amplitude associated with the action of $A_{v}^{k}$. The action of $A_{v}^{k}$ evolves the original vertex $v$ to a new vertex $v^{\prime}$, resulting in a new 4-simplex $0 v^{\prime} v 23$ and a new tetrahedron $v^{\prime} v 23$. According to bulk theory in the previous section, we must assign the 4 -simplex $0 v^{\prime} v 23$ a 4 -cocycle $\left[0 v^{\prime} v 23\right] \in H^{4}[G, \mathrm{U}(1)]$. A boundary vertex operator differs from a bulk vertex operator in the newly created temporal tetrahedra made of boundary vertices only, such the $v^{\prime} v 23$ in our example. Hence, for each such new temporal tetrahedron, we should assign a 3 -cochain in $C^{3}[K, \mathrm{U}(1)]$ as a factor in the corresponding amplitude, such as the 3-cochain $\left[v^{\prime} v 23\right]$ for the $v^{\prime} v 23$ in our example. The orientation of such 3-cochains follows our Convention 1. The amplitude $\mathcal{A}([0 v],[v 2],[23], k)$ now reads graphically and algebraically as

$$
\mathcal{A}([0 v],[v 2],[23], k)=
$$

where the $\cdots$ represents those 3 -cochains and 4-cocycles associated with those tetradedra and 4-simplexes not shown in the graph. Note that the amplitude of a bulk vertex operator should not contain any 3 -cochains because each bulk tetrahedron is shared by two 4simplexes and would contribute two 3-cochains that cancel out.

Next, we check whether the bulk vertex and face operators are still commuting projectors in ground states. Consider two boundary vertex operators $A_{v}^{k}$ and $A_{v^{\prime}}^{k^{\prime}}$. Obviously, if $v$ and $v^{\prime}$ are the two ends of an edge, $\left[A_{v}^{k}, A_{v^{\prime}}^{k^{\prime}}\right]=0$. What if $v$ and $v^{\prime}$ are connected by a boundary edge directly. Without loss of generality, Let us compute an example of such a commutator, i.e., assuming $v\left(v^{\prime}\right)=1(2)$ in the following.

$$
A_{1}^{k} A_{2}^{k^{\prime}}
$$

Then, $A_{1}^{k} A_{2}^{k^{\prime}}=A_{2}^{k^{\prime}} A_{1}^{k}$ requires that

$$
\frac{\left[12^{\prime} 245\right]\left[12^{\prime} 24\right]\left[1^{\prime} 12^{\prime} 35\right]\left[1^{\prime} 12^{\prime} 3\right]}{\left[12^{\prime} 235\right]\left[12^{\prime} 23\right]\left[1^{\prime} 12^{\prime} 45\right]\left[1^{\prime} 12^{\prime} 4\right]}=\frac{\left[1^{\prime} 2^{\prime} 245\right]\left[1^{\prime} 2^{\prime} 24\right]\left[1^{\prime} 1235\right]\left[1^{\prime} 123\right]}{\left[1^{\prime} 2^{\prime} 235\right]\left[1^{\prime} 2^{\prime} 23\right]\left[1^{\prime} 1245\right]\left[1^{\prime} 124\right]} .
$$

We apply the 4-cocycle condition

$$
\frac{\left[12^{\prime} 245\right]\left[1^{\prime} 1245\right]\left[1^{\prime} 12^{\prime} 25\right]}{\left[1^{\prime} 2^{\prime} 245\right]\left[1^{\prime} 12^{\prime} 45\right]\left[1^{\prime} 12^{\prime} 24\right]}=1, \frac{\left[12^{\prime} 235\right]\left[1^{\prime} 1235\right]\left[1^{\prime} 12^{\prime} 25\right]}{\left[1^{\prime} 2^{\prime} 235\right]\left[1^{\prime} 12^{\prime} 35\right]\left[1^{\prime} 12^{\prime} 23\right]}=1,
$$

to simplify eq. (3.8) and obtain the following constraint:

$$
\mathrm{d}\left[1^{\prime} 12^{\prime} 24\right]=\left[1^{\prime} 12^{\prime} 23\right] \mathrm{d}\left[1^{\prime} 12^{\prime} 23\right],
$$

where all the group variables involved take value in the subgroup $K$. 
Let us temporarily define a function $f: K^{4} \rightarrow \mathrm{U}(1)$ by $f(g, h, k, l)=[g, h, k, l] \mathrm{d}[g, h, k, l]$. Then, eq. (3.9) becomes $f\left(1^{\prime} 1,12^{\prime}, 2^{\prime} 2,24\right)=f\left(1^{\prime} 1,12^{\prime}, 2^{\prime} 2,23\right)$. Since the group elements [23] and [24] are arbitrary in $K$. We must have $f\left(1^{\prime} 1,12^{\prime}, 2^{\prime} 2, x\right)=c \forall x \in K$, where $c$ is a constant. By the normalization condition (2.3), however, $f\left(1^{\prime} 1,12^{\prime}, 2^{\prime} 2, x=1\right)=1$; hence, we must have $c=1$. In fact, the other group variables $\left[1^{\prime} 1\right],\left[12^{\prime}\right],\left[2^{\prime} 2\right]$ in eq. (3.9) are also arbitrary. We can conclude that $f(g, h, k, l) \equiv 1$. Since the example calculation of the commutator $\left[A_{v}^{k}, A_{v^{\prime}}^{k^{\prime}}\right]$ is generic, we would arrive at the same conclusion for the commutator on any two neighbouring boundary vertices $v$ and $v^{\prime}$. Therefore, requiring the commutativity of neighbouring boundary vertex operators leads to what we call the generalized Frobenius condition, ${ }^{4}$ which can be shown graphically as

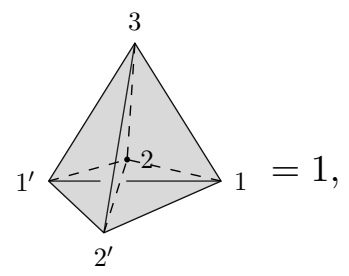

or algebraically as

$$
\mathrm{d}[g, h, k, l]=1, g, h, k, l \in K .
$$

The left hand side of eq. (3.10) is a 3 -complex $1^{\prime} 12^{\prime} 23$ due to the commutator $\left[A_{1}, A_{2}\right]$ and corresponds to the 4 -cocyle $[g, h, k, l]$ in eq. (3.11) because the group variables involved are arbitrary in $K$. The five tetrahedra composing $1^{\prime} 12^{\prime} 23$ produces five 3 -cochains, whose product corresponds to the 4 -coboundary $\mathrm{d}[g, h, k, l]$ in eq. (3.11). The generalized Frobenius condition (3.11) now can be understood as demanding that if the four group variables of a 4-cocycle are restricted in the subgroup $K$, it must be cohomologically trivial.

Nevertheless, the generalized Frobenius condition (3.11) is an equation that may not have a solution for certain $K \subseteq G$. If we would like our Hamiltonian being exactly solvable, i.e., all operators commuting, the generalized Frobenius condition must hold. If the Hamiltonian is not exactly solvable, we are not sure whether the boundary would be gapped or not. In this paper, we consider exactly solvable Hamiltonians only and thus enforce the generalized Frobenius condition.

Consequently, given a 4-cocycle $\omega \in H^{4}[G, \mathrm{U}(1)]$, a subgroup $K \subseteq G$ that allows solutions of the 3 -cochain $\alpha \in C^{3}[K, \mathrm{U}(1)]$ to the generalized Frobenius condition defines a class of physical boundary conditions, each for a solution. In the next section, we shall show that the set of solutions of $\alpha$ actually are in one-to-one correspondence with the 3 -cocyles in $H^{3}[K, \mathrm{U}(1)]$. Therefore, a permissible subgroup $K \subseteq G$ and a 3-cocycle $\tilde{\alpha} \in$ $H^{3}[K, \mathrm{U}(1)]$ define a physical boundary condition for a given bulk theory defined by $G$ and $\omega \in H^{4}[G, \mathrm{U}(1)]$.

Apart from the proof above, we need also to check the commutator between the boundary and bulk vertex operators and show that $A_{v}^{K}$ is a projector. These calculations would not result in any further constraints on our model. We thus leave them to appendix B.

\footnotetext{
${ }^{4}$ This is a generalization of the usual Frobenius condition [53].
} 


\section{One-to-one correspondence between 3-cochain and 3-cocycle}

We now prove the claim we made in the previous section that given a $K \subseteq G$, the 3-cochain solutions $\alpha \in C^{3}[K, \mathrm{U}(1)]$ to the generalized Frobenius condition (3.11) are in one-to-one correspondence with the 3-cocycles in $H^{3}[K, \mathrm{U}(1)]$.

Proof. Let us pick an arbitrary solution $\alpha_{0}$ of $\alpha$ to eq. (3.11). Denote $H^{3}[K, \mathrm{U}(1)]$ explicitly by $\left\{\tilde{\alpha}_{1}, \tilde{\alpha}_{2}, \ldots, \tilde{\alpha}_{n}\right\}$, where $\tilde{\alpha}_{i}$ and $\tilde{\alpha}_{j}$ are (representatives of) inequivalent 3 -cocycles. Because $\mathrm{d} \tilde{\alpha}_{i} \equiv 1$ and $\mathrm{d}\left(\alpha \tilde{\alpha}_{i}\right)=\mathrm{d} \alpha \mathrm{d} \tilde{\alpha}_{i}, \forall i, H^{3}[K, \mathrm{U}(1)]$ yields a set $\left\{\alpha_{0}, \alpha_{0} \tilde{\alpha}_{1}, \alpha_{0} \tilde{\alpha}_{2}, \ldots, \alpha_{0} \tilde{\alpha}_{n}\right\}$ of solutions to eq. (3.11). Here, the operator $d$ is the coboundary operator defined in appendix A.

Conversely, consider any solution $\alpha_{m}$ to eq. (3.11) other than $\alpha_{0}$, we have $\omega \mathrm{d} \alpha_{m}=$ $\omega \mathrm{d} \alpha_{0}=1$. Hence,

$$
\mathrm{d} \alpha_{m}=\mathrm{d} \alpha_{0} \Rightarrow \mathrm{d}\left(\alpha_{m} \alpha_{0}^{-1}\right)=1 \Rightarrow \alpha_{m}=\alpha_{0} \tilde{\alpha}_{m},
$$

where $\tilde{\alpha}_{m} \in H^{3}[K, U]$ is a 3 -cocycle. It does not matter which solution $\alpha_{0}$ we choose to generate the set of solutions $\left\{\alpha_{0}, \alpha_{0} \tilde{\alpha}_{1}, \alpha_{0} \tilde{\alpha}_{2}, \ldots, \alpha_{0} \tilde{\alpha}_{n}\right\} \stackrel{\text { def }}{=}\left\{\alpha_{i}|i=0, \ldots, n=| H^{3}[K, \mathrm{U}(1)] \mid-1\right\}$. For future convenience, we denote this set of 3 -cochains that specify all possible boundary conditions for a given $K \subseteq G$ by $\Lambda_{K}$. This establishes the claimed one-to-one correspondence.

Now the question is whether two pairs $(\omega, \alpha)$ and $\left(\omega^{\prime}, \alpha^{\prime}\right)$, where $\omega, \omega^{\prime} \in H^{4}[G, \mathrm{U}(1)]$ and $\alpha, \alpha^{\prime} \in \Lambda_{K}$, give rise to the same topological order with the same boundary condition. We shall report elsewhere the answer to this question. We refer the readers to ref. [63] for the answer to the version of this question for the TQD model of $2+1$-dimensional topological orders with boundaries.

\section{Ground-state wavefunctions on a 3-ball}

In this section, we derive the explicit ground-state wavefunctions of the TGT model on a 3-Ball.

Since here we are interested in ground states only, we can restrict our concern to the subspace $\mathcal{H}^{B_{f}=1}$ and the boundary degrees of freedom being all in certain subgroup $K \subseteq G$. Moreover, to guarantee the ground states, the bulk 4-cocycle $\omega$ and the boundary 3-cochain $\alpha$ must meet the generalized Frobenius condition (3.11).

Before deriving the formula of ground state wavefunction on a generic triangulation, let us consider the simplest triangulation of a 3-Ball figure 6. All the relevant degrees of freedom are on the boundary edges and thus take value in $K$. Flatness reduces the number of independent degrees of freedom to 3 , and we can choose them to be $h=[12], k=[23]$, $l=[34]$. In this case, the ground-state wavefunction $\Phi_{0}$ is simply the Dijkgraaf-Witten partition function with all the bulk degrees of freedom integrated out. So, the result is merely the 3-cocycle associated with the tetrahedron in figure 6. Namely,

$$
\Phi_{0}=[h, k, l],
$$

where $[h, k, l]=[1234]$ is the 3 -cocycle associated with the tetrahedron 1234 . 


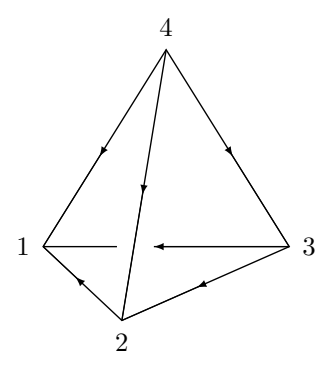

Figure 6. The simplest triangulation of a 3-ball.

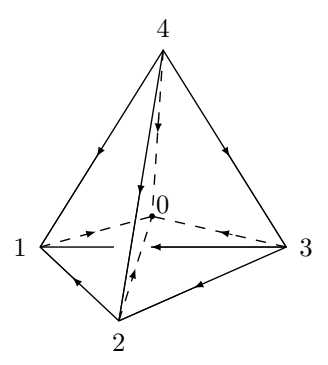

Figure 7. Another triangulation of a 3-ball.

Now we consider a more complicated configuration as illustrated in figure 7 with a bulk vertex 0. Instead of using Dijkgraaf-Witten partition function, we take a different strategy to obtain the ground-state wavefunction by finding the function that have an invariant form under the action of $A_{v}^{k}$ operators. This method is very effective for the special case where the vertices on the boundary bound a tetrahedron and are all connected to the single bulk vertex directly. Now the ground-state wavefunction consists of the associated 4-cocycle and 3-cochain:

$$
\Phi_{0}=[g, h, k, l][h, k, l]
$$

where $g=[01], h, k, l$ are previously defined, and $[g, h, k, l]=[01234]$ a 4 -cocycle.

It's easy to check that the wavefunction (5.2) is indeed invariant under the action of ground state projector $P^{0}$. For example, we check how (5.2) transforms under the action of $A_{1}^{x}$

$$
\begin{aligned}
A_{1}^{x} & {[g, h, k, l][h, k, l]|\Gamma\rangle } \\
& =\frac{[g \bar{x}, x, h, k][g \bar{x}, x, h k, l][x, h k, l][x, h, k]}{[g \bar{x}, x, h, k l][x, h, k l]}[g, h, k, l][h, k, l]|\Gamma\rangle \\
& =[x, h, k, l][g \bar{x}, x h, k, l] \frac{[x, h k, l][x, h, k][h, k, l]}{[x, h, k l]}|\Gamma\rangle \\
& =[g \bar{x}, x h, k, l][x h, k, l]\left|\Gamma^{\prime}\right\rangle \\
& =\left[g^{\prime}, h^{\prime}, k, l\right]\left[h^{\prime}, k, l\right]\left|\Gamma^{\prime}\right\rangle
\end{aligned}
$$




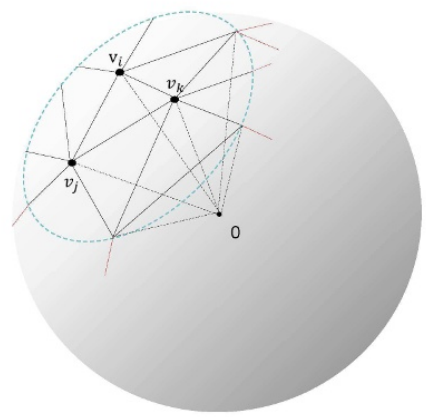

Figure 8. A general triangulation of a 3-ball.

where following 4-cocycle condition and generalized Frobenius condition are used.

$$
\begin{gathered}
\frac{[g \bar{x}, x, h, k][g \bar{x}, h k, l][g, h, k, l]}{[g \bar{x}, x, h, k l][x, h, k, l][g \bar{x}, x h, k, l]}=1 ; \\
{[x, h, k, l] \frac{[x, h k, l][x, h, k][h, k, l]}{[x, h, k l][x h, k, l]}=1 ;}
\end{gathered}
$$

We just discussed examples on two particular triangulations above. In the following we shall derive a generic formula for the ground state wavefunction on a triangulation as in figure 8 with one bulk vertex 0 and $N$ boundary vertices $v_{1}, v_{2}, \ldots v_{N}$. We will take the approach of Dijkgraaf-Witten partition functions. Imagine that the graph $\Gamma$ in figure 8 evolves from an initial graph $\Gamma^{\prime}$ where the bulk vertex is $0^{\prime}$, while the boundary vertices are the same. The ground-state wavefunction $\Phi_{0}(\Gamma)$ would be identified with the DijkgraafWitten partition function of the evolution from $\Gamma^{\prime}$ to $\Gamma$, in which the group element $\left[0^{\prime} 0\right]$ along the temporal edge $0^{\prime} 0$ is integrated out (i.e., summed over in our discrete case). Hence, $\Phi_{0}(\Gamma)$ should consist the 4-cocycles and 3-cochains associated with all the 3 -simplexes and tetrahedra due to the evolution, with $\left[0^{\prime} 0\right]$ summed over. The result is eq. (5.4).

$$
\Phi_{0}(\Gamma)=\sum_{\substack{x=\left[0^{\prime} 0\right] \in G \\ x a_{i} \in K}} \prod_{\left\{v_{i} v_{j} v_{k} \mid 2-\text { simplex }\right\}}\left[x, a_{i}, \bar{a}_{i} a_{j}, \bar{a}_{j} a_{k}\right]^{-\epsilon\left(0^{\prime} 0 v_{i} v_{j} v_{k}\right)}\left[x a_{i}, \bar{a}_{i} a_{j}, \bar{a}_{j} a_{k}\right]^{-\epsilon\left(0^{\prime} v_{i} v_{j} v_{k}\right)} .
$$

Here, the product runs over all boundary triangles $v_{i} v_{j} v_{k}$, and $a_{i}=\left[0 v_{i}\right]$. The signs $\epsilon\left(0^{\prime} 0 v_{i} v_{j} v_{k}\right)$ and $\epsilon\left(0^{\prime} v_{i} v_{j} v_{k}\right)$ follow the conventions defined before. For simplicity, we assume that $0^{\prime}$ and 0 are on the opposite sides of the boundary. The negative sign appearing in front of each $\epsilon$ results from our definition of ground-state wavefunction that $\Phi_{0}(\Gamma)=\left\langle\Gamma \mid \Phi_{0}\right\rangle$. It is straightforward to prove that $\Phi_{0}(\Gamma)$ is indeed a -1 eigenstate of the Hamiltonian (3.1). If $G=K$ and 4-cocycle is trivial, the ground-state wavefunction reduces to $\prod_{a_{i}, a_{j}, a_{k}}\left[a_{i}, \bar{a}_{i} a_{j}, \bar{a}_{j} a_{k}\right]^{\epsilon\left(0 v_{i} v_{j} v_{k}\right)}$.

In the cases where there are more than one bulk vertices, the ground-state wavefunction can be simply obtained from $\Phi_{0}(\Gamma)$ by performing bulk Pachner moves, which would modify the 4-cocyles of $\Phi_{0}(\Gamma)$ only. We refer the reader to ref. [21] for the precise algebraic forms of the 3-dimensional Pachner moves. 


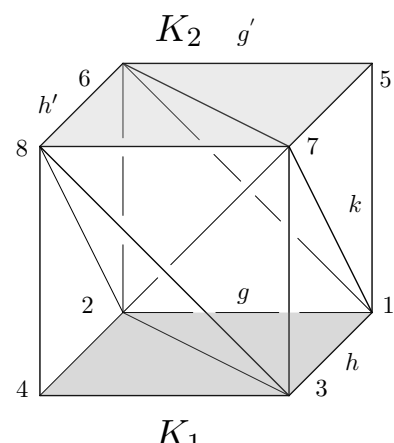

Figure 9. A simple triangulation of a 3-cylinder. Vertex ordering is obvious, and arrows are thus omitted. The surface 5678 and 1234 are the two boundaries. We let $[12]=[34]=g,[24]=[13]=$ $h,[56]=[78]=g^{\prime},[57]=[68]=h^{\prime},[15]=[26]=[37]=[48]=k$.

\section{GSD on a 3-cylinder}

On a 3-ball, the fundamental group of boundary and bulk are both trivial. So the GSD of our model on a 3 -ball is one. To see how boundary conditions affect the GSD of a $(3+1)$ dimensional topological order, let us consider our model defined on a simple triangulation (see figure 9) of a 3-cylinder (see figure 4), where the top and the bottom surfaces are the two boundaries with subgroups $K_{2}, K_{1} \subseteq G$.

We let $g \in K_{1}$ and $g^{\prime} \in K_{2}$, but $k \in G$. Since we are interested in ground states only, we let all the triangles in figure 9 be flat, i.e., we are working in the subspace $\mathcal{H}^{B_{f}=1}$. Consequently, we have $h^{\prime}=\bar{k} h k$ and $g^{\prime}=\bar{k} g k$.

Note that there are only two boundary vertices in figure 9 , namely, vertices $1,2,3$, and 4 are identified, and vertices 5, 6, 7, and 8 are identified. The ground-state projector then reads

$$
P_{3 \text {-cyl }}^{0}=\prod_{v \in \partial \Gamma} A_{v}^{K}=\frac{1}{\left|K_{1}\right|\left|K_{2}\right|} \sum_{x \in K_{1}} A_{1=2=3=4}^{x} \sum_{y \in K_{2}} A_{5=6=7=8}^{y} .
$$

Here is an important technical remark. In acting the above operator on the 3-cylinder, An operator $A^{x}$ must act on vertices $1,2,3$, and 4 individually, as if these vertices are different; the identification of $1,2,3$, and 4 will be automatically accounted for by the periodic boundary condition and there is only one normalization factor $\frac{1}{\left|K_{1}\right|}$. The same procedure applies to $A^{y}$ on vertices $5,6,7$, and 8 . The GSD on a 3 -cylinder is then the trace of $P_{3 \text {-cyl }}^{0}$ :

$$
G S D_{3 \text {-cyl }}=\operatorname{Tr}_{\mathcal{H}^{B_{f}}=1} P_{3 \text {-cyl }}^{0}=\sum_{\substack{h, g \in K_{1}, h^{\prime}, g^{\prime} \in K_{2}, k \in G}} \delta_{g^{\prime}, \bar{k} g k} \delta_{h^{\prime}, \bar{k} h k}
$$

We present the final closed-form of the GSD on a 3-cylinder as follows but leave the tedious derivation to appendix $\mathrm{C}$.

$$
G S D_{3-\mathrm{cyl}}=\sum_{\substack{x, h, g \in K_{1} \\ y, h^{\prime}, g^{\prime} \in K_{2}}} \sum_{k \in G} \frac{\delta_{g^{\prime}, \bar{k} g k} \delta_{h^{\prime}, \bar{k} h k} \delta_{g, x g \bar{x}} \delta_{h, x h \bar{x}} \delta_{k, x k \bar{y}}}{\left|K_{1}\right|\left|K_{2}\right|} \frac{[\bar{x}, k]_{h \bar{g}, g}}{[k, \bar{y}]_{h \bar{g}, g}} \frac{\eta^{g}(h \bar{g}, x)}{\eta^{\bar{k} g k}(\bar{k} h \bar{g} k, y)},
$$


where $[a, b]_{m, n}=\frac{[m, a, b]_{n}[a, b, \overline{(a b)} m a b]_{n}}{[a, \bar{a} m a, b]_{n}}$ is a doubly-twisted 2-cocycle, and $\eta^{n}(m, a)$ is defined by $\frac{[\bar{a}, m]_{n}}{[m, \bar{a}]_{n}}$ with $[\bar{a}, m]_{n}$ being a twisted 2-cochain. The factor $\frac{\eta^{g}(h \bar{g}, x)}{\eta^{k g k}(\bar{k} h \bar{g} k, y)}$ can be rewritten in more compactly as $\frac{1}{\eta^{g, h \bar{g}}(\bar{x}, \bar{k})}$, which is trivial when the doubly twisted 2-cocycle is cohomologically trivial.

This result generalizes the GSD of the $(2+1)$-dimensional TQD model on a cylinder. It's easy to see that when $G$ is an Abelian group, The cochain term is simply 1 .

\section{Examples: $G=\mathbb{Z}_{2} \times \mathbb{Z}_{2} \times \mathbb{Z}_{2}$}

Here we consider the TGT model with the gauge group $G=\mathbb{Z}_{2} \times \mathbb{Z}_{2} \times \mathbb{Z}_{2}$ as an example. We check for each subgroup of $G$ the solutions to the generalized Frobenius condition (3.11). Note that TGT model with this $G$ yields only Abelian excitations [21]. The 4-th cohomology group of $G$ is $H^{4}\left[\mathbb{Z}_{2} \times \mathbb{Z}_{2} \times \mathbb{Z}_{2}, \mathrm{U}(1)\right]=\mathbb{Z}_{2}^{2} \times \mathbb{Z}_{2}^{2} \times \mathbb{Z}_{2}^{2} \times \mathbb{Z}_{2}^{2}$.

The generators of the 4-cocycles $\omega$ are (here $(i, j)=(1,2),(2,3)$ or $(1,3)$ and $(i, j, l)=(1,2,3))$ :

$$
\begin{aligned}
\omega_{\mathrm{II}}^{(1 s t, i j)}(a, b, c, d) & =e^{\left(\frac{\pi \mathrm{i}}{2}\left(a_{i} b_{j}\right)\left(c_{j}+d_{j}-\left[c_{j}+d_{j}\right]\right)\right)}, \\
\omega_{\mathrm{II}}^{(2 n d, i j)}(a, b, c, d) & =e^{\left(\frac{\pi \mathrm{i}}{2}\left(a_{j} b_{i}\right)\left(c_{i}+d_{i}-\left[c_{i}+d_{i}\right]\right)\right)}, \\
\omega_{\mathrm{III}}^{(1 s t, i j l)}(a, b, c, d) & =e^{\left(\frac{\pi \mathrm{i}}{2}\left(a_{i} b_{j}\right)\left(c_{l}+d_{l}-\left[c_{l}+d_{l}\right]\right)\right)}, \\
\omega_{\mathrm{III}}^{(2 n d, i j l)}(a, b, c, d) & =e^{\left(\frac{\pi \mathrm{i}}{2}\left(a_{l} b_{i}\right)\left(c_{j}+d_{j}-\left[c_{j}+d_{j}\right]\right)\right)},
\end{aligned}
$$

where $[x]=x \bmod 2$. Any 4 -cocycle $\omega$ can be written in terms of the above generators. For example:

$$
\begin{aligned}
\omega & =\prod_{\substack{(i, j)=(1,2),(2,3),(1,3) \\
(i, j, l)=(1,2,3)}} \omega_{\mathrm{II}}^{(1 s t, i j)} \omega_{\mathrm{II}}^{(2 n d, i j)} \omega_{\mathrm{III}}^{(1 s t, i j l)} \omega_{\mathrm{III}}^{(2 n d, i j l)} \\
& =\prod_{\substack{(i, j)=(1,2),(2,3),(1,3) \\
(i, j, l)=(1,2,3)}}\left(\omega_{\mathrm{II}}^{(1 s t, i j)}\right)^{p_{\mathrm{II}(i j)}^{(1 s t)}}\left(\omega_{\mathrm{II}}^{(2 n d, i j)}\right)^{p_{\mathrm{II}(i j)}^{(2 n d)}}\left(\omega_{\mathrm{III}}^{(1 s t, i j l)}\right)^{p_{\mathrm{III}(i j l)}^{(1 s t)}}\left(\omega_{\mathrm{III}}^{(2 n d, i j l)}\right)^{p_{\mathrm{III}(i j l)}^{(2 n d)}}
\end{aligned}
$$

where $p_{\mathrm{II}(i j)}^{(1 s t)}, p_{\mathrm{II}(i j)}^{(2 n d)}, p_{\mathrm{III}(i j l)}^{(1 s t)}, p_{\mathrm{III}(i j l)}^{(2 n d)} \in \mathbb{Z}_{2}$ are called the topological indices [20, 21]. Table 1 then records the 3 -cochain solutions to the generalized Frobenius condition eq. (3.11) for each generator of the 4-cocycles and each subgroup $K \subseteq G$.

\section{Acknowledgments}

This work is supported by the Shanghai Pujiang Program No. KBH1512328. 


\begin{tabular}{|c|c|c|c|c|c|c|c|c|c|}
\hline$K$ & $\omega_{0}$ & $\omega_{I I}^{(1 s t, 12)}$ & $\omega_{I I}^{(1 s t, 23)}$ & $\omega_{I I}^{(1 s t, 13)}$ & $\omega_{I I}^{(2 n d, 12)}$ & $\omega_{I I}^{(2 n d, 23)}$ & $\omega_{I I}^{(2 n d, 13)}$ & $\omega_{I I I}^{(1 s t, 123)}$ & $\omega_{I I I}^{(2 n d, 123)}$ \\
\hline$\{000\}$ & 1 & 1 & 1 & 1 & 1 & 1 & 1 & 1 & 1 \\
\hline$\{000,001\}=\mathbb{Z}_{2}$ & $\mathbb{Z}_{2}$ & $\mathbb{Z}_{2}$ & $\mathbb{Z}_{2}$ & $\mathbb{Z}_{2}$ & $\mathbb{Z}_{2}$ & $\mathbb{Z}_{2}$ & $\mathbb{Z}_{2}$ & $\mathbb{Z}_{2}$ & $\mathbb{Z}_{2}$ \\
\hline$\{000,010\}=\mathbb{Z}_{2}$ & $\mathbb{Z}_{2}$ & $\mathbb{Z}_{2}$ & $\mathbb{Z}_{2}$ & $\mathbb{Z}_{2}$ & $\mathbb{Z}_{2}$ & $\mathbb{Z}_{2}$ & $\mathbb{Z}_{2}$ & $\mathbb{Z}_{2}$ & $\mathbb{Z}_{2}$ \\
\hline$\{000,100\}=\mathbb{Z}_{2}$ & $\mathbb{Z}_{2}$ & $\mathbb{Z}_{2}$ & $\mathbb{Z}_{2}$ & $\mathbb{Z}_{2}$ & $\mathbb{Z}_{2}$ & $\mathbb{Z}_{2}$ & $\mathbb{Z}_{2}$ & $\mathbb{Z}_{2}$ & $\mathbb{Z}_{2}$ \\
\hline$\{000,011,001,010\}=\mathbb{Z}_{2} \times \mathbb{Z}_{2}$ & $\mathbb{Z}_{2}^{3}$ & $\mathbb{Z}_{2}^{3}$ & $\times$ & $\mathbb{Z}_{2}^{3}$ & $\mathbb{Z}_{2}^{3}$ & $\times$ & $\mathbb{Z}_{2}^{3}$ & $\mathbb{Z}_{2}^{3}$ & $\mathbb{Z}_{2}^{3}$ \\
\hline$\{000,101,001,100\}=\mathbb{Z}_{2} \times \mathbb{Z}_{2}$ & $\mathbb{Z}_{2}^{3}$ & $\mathbb{Z}_{2}^{3}$ & $\mathbb{Z}_{2}^{3}$ & $\times$ & $\mathbb{Z}_{2}^{3}$ & $\mathbb{Z}_{2}^{3}$ & $\times$ & $\mathbb{Z}_{2}^{3}$ & $\mathbb{Z}_{2}^{3}$ \\
\hline$\{000,110,100,010\}=\mathbb{Z}_{2} \times \mathbb{Z}_{2}$ & $\mathbb{Z}_{2}^{3}$ & $\times$ & $\mathbb{Z}_{2}^{3}$ & $\mathbb{Z}_{2}^{3}$ & $\times$ & $\mathbb{Z}_{2}^{3}$ & $\mathbb{Z}_{2}^{3}$ & $\mathbb{Z}_{2}^{3}$ & $\mathbb{Z}_{2}^{3}$ \\
\hline $\mathbb{Z}_{2} \times \mathbb{Z}_{2} \times \mathbb{Z}_{2}$ & $\mathbb{Z}_{2}^{7}$ & $\times$ & $\times$ & $\times$ & $\times$ & $\times$ & $\times$ & $\times$ & $\times$ \\
\hline
\end{tabular}

Table 1. 3-cochain solutions to the generalized Frobenius condition for $G=\mathbb{Z}_{2} \times \mathbb{Z}_{2} \times \mathbb{Z}_{2}$. A big $\times$ indicates "no solution".

\section{A A brief introduction to cohomology groups $H^{n}[G, \mathrm{U}(1)]$}

We record a few necessary ingredients of the cohomology groups $H^{n}[G, \mathrm{U}(1)]$ of finite groups $G$ in this appendix.

The $n$-th cochain group $C^{n}[G, \mathrm{U}(1)]$ of $G$ is an Abelian group of $n$-cochains, i.e., functions $c\left(g_{1}, \ldots, g_{n}\right): G^{\times n} \rightarrow \mathrm{U}(1)$, where $g_{i} \in G$. The group product reads $c\left(g_{1}, \ldots, g_{n}\right) c^{\prime}\left(g_{1}, \ldots, g_{n}\right)=\left(c c^{\prime}\right)\left(g_{1}, \ldots, g_{n}\right)$. The coboundary operator d maps $C^{n}$ to $C^{n+1}$, i.e,

$$
\begin{aligned}
\mathrm{d} & : C^{n} \rightarrow C^{n+1} \\
& : c\left(g_{1}, \ldots, g_{n}\right) \mapsto(\mathrm{d} c)\left(g_{0}, g_{1} \ldots, g_{n}\right),
\end{aligned}
$$

where

$$
(\mathrm{d} c)\left(g_{0}, g_{1} \ldots, g_{n}\right)=\prod_{i=0}^{n+1} c\left(\ldots, g_{i-2}, g_{i-1} g_{i}, g_{i+1}, \ldots\right)^{(-1)^{i}} .
$$

The series of variables starts at $g_{0}$, and ends at $g_{n-1}$. Equation (2.4) offers the example for $n=4$. The nilpotency of $\mathrm{d}$, i.e., $\mathrm{d}^{2} c=1$, leads to the following exact sequence:

$$
\cdots C^{n-1} \stackrel{\mathrm{d}}{\rightarrow} C^{n} \stackrel{\mathrm{d}}{\rightarrow} C^{n+1} \ldots .
$$

The images of the coboundary operator, $\operatorname{im}\left(\mathrm{d}: C^{n-1} \rightarrow C^{n}\right)$, form the $n$-th coboundary group, whose elements are dubbed $n$-coboundaries. The kernel $\operatorname{ker}\left(\mathrm{d}: C^{n} \rightarrow C^{n+1}\right)$ is the $n$-cocycle group, whose elements are the $n$-cochains satisfying the cocycle condition $\mathrm{d} c=1$. Equation (2.4) is the example for $n=4$. The definition of the $n$-th cohomology group is the quotient group that follows from the exact sequence (A.1):

$$
H^{n}[G, \mathrm{U}(1)]:=\frac{\operatorname{ker}\left(\mathrm{d}: C^{n} \rightarrow C^{n+1}\right)}{\operatorname{im}\left(\mathrm{d}: C^{n-1} \rightarrow C^{n}\right)} .
$$

The group $H^{n}[G, \mathrm{U}(1)]$ is Abelian by construction and comprises the equivalence classes of the $n$-cocyles that differ from one another by merely an $n$-coboundary. A trivial $n$-cocycle is one that can be written as a $n$-coboundary. 


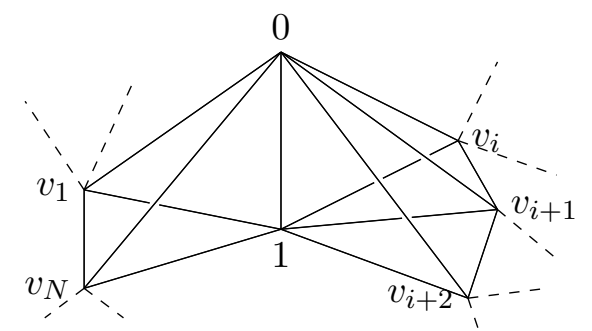

Figure 10. A portion of a graph, where some boundary vertices $\left\{1, v_{1}, v_{2}, \ldots, v_{N}\right\}$ and a bulk vertex 0 are shown. We assume that $v_{i+1}>v_{i}>1$.

One can define a slant product that maps $c_{g}$ as follows.

$$
\begin{aligned}
& c_{g}\left(g_{1}, g_{2}, \ldots, g_{n-1}\right) \\
& =c\left(g, g_{1}, g_{2}, \ldots, g_{n-1}\right)^{(-1)^{n-1}} \prod_{j=1}^{n-1} c\left(g_{1}, \ldots, g_{j},\left(g_{1} \cdots g_{j}\right)^{-1} g\left(g_{1} \cdots g_{j}\right), \ldots, g_{n-1}\right)^{(-1)^{n-1+j}} .
\end{aligned}
$$

The twisted 3-cocycles and the doubly-twisted 2-cocycles we have encountered in the main text are examples of this slant product.

\section{B Some properties about $A_{v}^{K}$ operators}

First, let us prove that $A_{v}^{K}$ is a projector. To this end, we need to show that $A_{v}^{k^{\prime}} A_{v}^{k}=A_{v}^{k^{\prime} k}$. Consider a portion (figure 10) of a graph $\Gamma$.

We denote the state vector associated with the graph $\Gamma$ by $|\Gamma\rangle$. Without loss of generality, we consider the action of $A_{v}^{K}$ on vertex 1 . The action of $A_{1}^{k}$ on vertex 1 would replace vertex 1 by a new vertex $1^{\prime}$, with $1^{\prime}<1$. Then, acting $A_{v}^{K}$ twice on vertex 1 involves terms like $A_{1^{\prime}}^{k^{\prime}} A_{1}^{k}$ acting on $|\Gamma\rangle$. In the end, $\Gamma$ would become some $\Gamma^{\prime \prime}$, where vertex 1 becomes vertex $1^{\prime \prime}$. We have

$$
\begin{aligned}
A_{1^{\prime}}^{k^{\prime}} A_{1}^{k}|\Gamma\rangle= & \delta_{\left[1^{\prime} 1\right], k} \delta_{\left[1^{\prime \prime} 1^{\prime}\right], k^{\prime}}\left[01^{\prime} 1 v_{1} v_{N}\right]\left[1^{\prime} 1 v_{1} v_{N}\right]\left[01^{\prime \prime} 1^{\prime} v_{1} v_{N}\right]\left[1^{\prime \prime} 1^{\prime} v_{1 v_{N}}\right] \\
& \times \prod_{i=1}^{N} \frac{1}{\left[01^{\prime} 1 v_{i} v_{i+1}\right]\left[1^{\prime} 1 v_{i} v_{i+1}\right]\left[01^{\prime \prime} 1^{\prime} v_{i} v_{i+1}\right]\left[1^{\prime \prime} 1^{\prime} v_{i} v_{i+1}\right]}\left|\Gamma^{\prime \prime}\right\rangle,
\end{aligned}
$$

Using the following instances of the 4-cocycle condition (2.4) and the generalized Frobenius condition (3.11)

$$
\begin{array}{r}
\frac{\left[1^{\prime \prime} 1^{\prime} 1 v_{i} v_{i+1}\right]\left[01^{\prime \prime} 1 v_{i} v_{i+1}\right]\left[01^{\prime \prime} 1^{\prime} 1 v_{i+1}\right]}{\left[01^{\prime} 1 v_{i} v_{i+1}\right]\left[01^{\prime \prime} 1^{\prime} v_{i} v_{i+1}\right]\left[01^{\prime \prime} 1^{\prime} 1 v_{i}\right]}=1, \\
\frac{\left[1^{\prime \prime} 1^{\prime} 1 v_{1} v_{N}\right]\left[01^{\prime \prime} 1 v_{1} v_{N}\right]\left[01^{\prime \prime} 1^{\prime} 1 v_{N}\right]}{\left[01^{\prime} 1 v_{1} v_{N}\right]\left[01^{\prime \prime} 1^{\prime} v_{1} v_{N}\right]\left[01^{\prime \prime} 1^{\prime} 1 v_{1}\right]}=1, \\
{\left[1^{\prime \prime} 1^{\prime} 1 v_{i} v_{i+1}\right] \frac{\left[1^{\prime} 1 v_{i} v_{i+1}\right]\left[1^{\prime \prime} 1^{\prime} v_{i} v_{i+1}\right]\left[1^{\prime \prime} 1^{\prime} 1 v_{i}\right]}{\left[1^{\prime \prime} 1 v_{i} v_{i+1}\right]\left[1^{\prime \prime} 1^{\prime} 1 v_{i+1}\right]}=1,} \\
{\left[1^{\prime \prime} 1^{\prime} 1 v_{1} v_{N}\right] \frac{\left[1^{\prime} 1 v_{1} v_{N}\right]\left[1^{\prime \prime} 1^{\prime} v_{1} v_{N}\right]\left[1^{\prime \prime} 1^{\prime} 1 v_{1}\right]}{\left[1^{\prime \prime} 1 v_{1} v_{N}\right]\left[1^{\prime \prime} 1^{\prime} 1 v_{N}\right]}=1,}
\end{array}
$$


where $i=1,2 \ldots N$, eq. (B.1) becomes

$$
\begin{aligned}
A_{1^{\prime}}^{k^{\prime}} A_{1}^{k}|\Gamma\rangle= & \delta_{\left[1^{\prime} 1\right], k} \delta_{\left[1^{\prime \prime} 1^{\prime}\right], k^{\prime}}\left[01^{\prime \prime} 1 v_{1} v_{N}\right]\left[1^{\prime \prime} 1 v_{1} v_{N}\right] \prod_{i=1}^{N} \frac{1}{\left[01^{\prime \prime} 1 v_{i} v_{i+1}\right]\left[1^{\prime \prime} 1 v_{i} v_{i+1}\right]} \frac{\left[01^{\prime \prime} 1^{\prime} 1 v_{N}\right]}{\left[01^{\prime \prime} 1^{\prime} 1 v_{1}\right]} \\
& \times \prod_{i=1}^{N} \frac{\left[01^{\prime \prime} 1^{\prime} 1 v_{i}\right]}{\left[01^{\prime \prime} 1^{\prime} 1 v_{i+1}\right]} \frac{\left[1^{\prime \prime} 1^{\prime} 1 v_{N}\right]}{\left[1^{\prime \prime} 1^{\prime} 1 v_{1}\right]} \prod_{i=1}^{N} \frac{\left[1^{\prime \prime} 1^{\prime} 1 v_{i}\right]}{\left[1^{\prime \prime} 1^{\prime} 1 v_{i+1}\right]}\left|\Gamma^{\prime \prime}\right\rangle \\
= & \delta_{\left[1^{\prime} 1\right], k} \delta_{\left[1^{\prime \prime} 1^{\prime}\right], k^{\prime}}\left[01^{\prime \prime} 1 v_{1} v_{N}\right]\left[1^{\prime \prime} 1 v_{1} v_{N}\right] \prod_{i=1}^{N} \frac{1}{\left[01^{\prime \prime} 1 v_{i} v_{i+1}\right]\left[1^{\prime \prime} 1 v_{i} v_{i+1}\right]}\left|\Gamma^{\prime \prime}\right\rangle=A_{1}^{k^{\prime} k}|\Gamma\rangle .
\end{aligned}
$$

Since $A_{v}^{K}$ is an average of $A_{v}^{k}$ over $K$, by the rearrangement lemma of group theory, $\left(A_{v}^{K}\right)^{2}=A_{v}^{K}$. Thus, $A_{v}^{K}$ is a projector. One may ask if there are more than one bulk vertices in this graph, would eq. (B.2) still hold? The answer is yes, as from eq. (B.2), we see that the 4-cocycle terms and 3-cochain terms are decoupled, the effect of $A_{v}^{k^{\prime}} A_{v}^{k}$ on the tetrahedra and faces around vertex 1 will always leads to 4 -cocyles and 3-cochains that can cancel out by applying the 4-cocycle condition and generalized Frobenius condition, hence arriving at the last equality in eq. (B.2).

Consider again figure 10, we can also prove the commutativity of bulk and boundary vertex operators. The vanishing of $\left[A_{0}^{g}, A_{1}^{k}\right]$ requires

$$
\frac{\left[01^{\prime} 1 v_{i} v_{N}\right]}{\left[0^{\prime} 01^{\prime} v_{1} v_{N}\right]} \prod_{i=1}^{N} \frac{\left[0^{\prime} 01^{\prime} v_{i} v_{j}\right]}{\left[01^{\prime} 1 v_{i} v_{j}\right]}=\frac{\left[0^{\prime} 1^{\prime} 1 v_{1} v_{N}\right]}{\left[0^{\prime} 01 v_{1} v_{N}\right]} \prod_{i=1}^{N} \frac{\left[0^{\prime} 01 v_{i} v_{j}\right]}{\left[0^{\prime} 1^{\prime} 1 v_{i} v_{j}\right]}
$$

The 3-cochains on both sides of the equation cancel and are thus neglected. Using the following instances of the 4-cocycle condition (2.4)

$$
\frac{\left[01^{\prime} 1 v_{i} v_{i+1}\right]\left[0^{\prime} 01 v_{i} v_{i+1}\right]\left[0^{\prime} 01^{\prime} 1 v_{i+1}\right]}{\left[0^{\prime} 1^{\prime} 1 v_{i} v_{i+1}\right]\left[0^{\prime} 01^{\prime} v_{i} v_{i+1}\right]\left[0^{\prime} 01^{\prime} 1 v_{i}\right]}=1, \frac{\left[01^{\prime} 1 v_{1} v_{N}\right]\left[0^{\prime} 01 v_{1} v_{N}\right]\left[0^{\prime} 01^{\prime} 1 v_{N}\right]}{\left[0^{\prime} 1^{\prime} 1 v_{1} v_{N}\right]\left[0^{\prime} 01^{\prime} v_{1} v_{N}\right]\left[0^{\prime} 01^{\prime} 1 v_{1}\right]}=1
$$

eq. (B.3) turns out to be

$$
\frac{\left[0^{\prime} 01^{\prime} 1 v_{N}\right]}{\left[0^{\prime} 01^{\prime} 1 v_{1}\right]} \prod_{i=1}^{N-1} \frac{\left[0^{\prime} 01^{\prime} 1 v_{i}\right]}{\left[0^{\prime} 01^{\prime} 1 v_{i+1}\right]}=1
$$

which is an identity because all the factors cancel out. Note that when $v^{\prime}$ and $v$ do not connect directly, $\left[A_{v}^{g}, A_{v^{\prime}}^{k}\right]=0$ is obvious. Together with the proof above, we conclude that bulk and boundary vertex operators commute in the subspace $\mathcal{H}^{B_{f}=1}$.

\section{The derivation of GSD on a 3-cylinder}

We derive eq. (6.3) in this appendix. We first check how the projector acts on the 3-cylinder. To simply the calculation, however, we choose to act the vertex operators involved in the 
ground-state projector (6.1) on the vertices in figure 9 in descending order. We have

$$
\begin{aligned}
& P_{3 \text {-cyl }}^{0}|12,13,15,56,57\rangle=\frac{1}{\left|K_{1}\right|\left|K_{2}\right|} \sum_{x \in K_{1}} \sum_{y \in K_{2}}\left|1^{\prime} 2^{\prime}, 1^{\prime} 3^{\prime}, 1^{\prime} 5^{\prime}, 5^{\prime} 6^{\prime}, 5^{\prime} 7^{\prime}\right\rangle \\
& \quad \times \frac{\left[2348^{\prime} 8\right]\left[2678^{\prime} 8\right]}{\left[2378^{\prime} 8\right]} \frac{\left[678^{\prime} 8\right]\left[268^{\prime} 8\right]\left[348^{\prime} 8\right]}{\left[248^{\prime} 8\right]\left[378^{\prime} 8\right]} \frac{\left[237^{\prime} 78^{\prime}\right]\left[1267^{\prime} 7\right]}{\left[1237^{\prime} 7\right]\left[267^{\prime} 78^{\prime}\right]\left[1567^{\prime} 7\right]} \frac{\left[137^{\prime} 7\right]\left[37^{\prime} 78^{\prime}\right]}{\left[157^{\prime} 7\right]\left[567^{\prime} 7\right]\left[67^{\prime} 78^{\prime}\right]} \\
& \times \frac{\left[156^{\prime} 67^{\prime}\right]\left[26^{\prime} 67^{\prime} 8^{\prime}\right]}{\left[126^{\prime} 67^{\prime}\right]} \frac{\left[56^{\prime} 67^{\prime}\right]\left[6^{\prime} 67^{\prime} 8^{\prime}\right]\left[156^{\prime} 6\right]}{\left[26^{\prime} 68^{\prime}\right]\left[126^{\prime} 6\right]} \frac{1}{\left[15^{\prime} 56^{\prime} 7^{\prime}\right]} \frac{\left[15^{\prime} 57^{\prime}\right]}{\left[15^{\prime} 56^{\prime}\right]\left[5^{\prime} 56^{\prime} 7^{\prime}\right]} \frac{1}{\left[234^{\prime} 48^{\prime}\right]} \frac{\left[24^{\prime} 48^{\prime}\right]}{\left[234^{\prime} 4\right]\left[34^{\prime} 48^{\prime}\right]} \\
& \quad \times \frac{\left[23^{\prime} 34^{\prime} 8^{\prime}\right]\left[123^{\prime} 37^{\prime}\right]}{\left[23^{\prime} 37^{\prime} 8^{\prime}\right]} \frac{\left[123^{\prime} 3\right]\left[23^{\prime} 34^{\prime}\right]\left[3^{\prime} 34^{\prime} 8^{\prime}\right]}{\left[13^{\prime} 37^{\prime}\right]\left[3^{\prime} 37^{\prime} 8^{\prime}\right]} \frac{\left[2^{\prime} 23^{\prime} 7^{\prime} 8^{\prime}\right]\left[12^{\prime} 26^{\prime} 7^{\prime}\right]}{\left[2^{\prime} 23^{\prime} 4^{\prime} 8^{\prime}\right]\left[12^{\prime} 23^{\prime} 7^{\prime}\right]\left[2^{\prime} 26^{\prime} 7^{\prime} 8^{\prime}\right]} \frac{\left[12^{\prime} 26^{\prime}\right]\left[2^{\prime} 26^{\prime} 8^{\prime}\right]}{\left[12^{\prime} 23^{\prime}\right]\left[2^{\prime} 23^{\prime} 4^{\prime}\right]\left[2^{\prime} 24^{\prime} 8^{\prime}\right]} \\
& \quad \times \frac{\left[1^{\prime} 15^{\prime} 6^{\prime} 7^{\prime}\right]\left[1^{\prime} 12^{\prime} 3^{\prime} 7^{\prime}\right]}{\left[1^{\prime} 12^{\prime} 6^{\prime} 7^{\prime}\right]} \frac{\left[1^{\prime} 13^{\prime} 7^{\prime}\right]\left[1^{\prime} 12^{\prime} 3^{\prime}\right]\left[1^{\prime} 15^{\prime} 6^{\prime}\right]}{\left[1^{\prime} 15^{\prime} 7^{\prime}\right]\left[1^{\prime} 12^{\prime} 6^{\prime}\right]} .
\end{aligned}
$$

By renaming of variables, $[12]=[34]=g,[24]=[13]=h,[56]=[78]=g^{\prime},[57]=[68]=h^{\prime}$, $[15]=[26]=[37]=[48]=k$, we have

$$
\begin{aligned}
& P_{3-\mathrm{cyl}}^{0}\left|g, h, k, g^{\prime}, h^{\prime}\right\rangle=\frac{1}{\left|K_{1}\right|\left|K_{2}\right|} \sum_{x \in K_{1}} \sum_{y \in K_{2}}\left|x g \bar{x}, x h \bar{x}, x k \bar{y}, y g^{\prime} \bar{y}, y h^{\prime} \bar{y}\right\rangle \\
& \quad \times \frac{[h \bar{y}, g, k \bar{y}, y]\left[k, h^{\prime} \bar{g}^{\prime}, g^{\prime} \bar{y}, y\right]\left[h \bar{g}, k \bar{y}, y, g^{\prime} \bar{y}\right]\left[g, k, h^{\prime} \bar{g}^{\prime} \bar{y}, y\right]\left[k, g^{\prime} \bar{y}, y, h^{\prime} \bar{g}^{\prime} \bar{y}\right]\left[k \bar{y}, y, h^{\prime} \bar{g}^{\prime} \bar{y}, y g^{\prime} \bar{y}\right]}{\left[h \bar{y}, k, g^{\prime} \bar{y}, y\right][g, h \bar{y}, k \bar{y}, y]\left[k, h^{\prime} \bar{g}^{\prime} \bar{y}, y, g^{\prime} \bar{y}\right]\left[k, g^{\prime}, h^{\prime} \bar{g}^{\prime} \bar{y}, y\right]\left[g, k \bar{y}, y, h^{\prime} \bar{g}^{\prime} \bar{y}\right]\left[k \bar{y}, y, g^{\prime} \bar{y}, y h^{\prime} \bar{g}^{\prime} \bar{y}\right]} \\
& \quad \times \frac{[h \bar{y} \bar{x}, x, g \bar{x}, x k \bar{y}][g, h \bar{y} \bar{x}, x, k \bar{y}]\left[x, h \bar{y} \bar{x}, x k \bar{y}, y g^{\prime} \bar{y}\right]\left[g \bar{x}, x, k \bar{y}, y h^{\prime} \bar{g}^{\prime} \bar{y}\right]\left[x, k \bar{y}, y g^{\prime} \bar{y}, y h^{\prime} \bar{g}^{\prime} \bar{y}\right][x, g \bar{x}, x h \bar{y} \bar{x}, x k \bar{y}]}{[h \bar{y}, g \bar{x}, x, k \bar{y}]\left[h \bar{y} \bar{x}, x, k \bar{y}, y g^{\prime} \bar{y}\right][x, h \bar{y} \bar{x}, x g \bar{x}, x k \bar{y}][g \bar{x}, x, h \bar{y} \bar{x}, x k \bar{y}]\left[x, k \bar{y}, y h^{\prime} \bar{g}^{\prime} \bar{y}, y g^{\prime} \bar{y}\right]\left[x, g \bar{x}, x k \bar{y}, y h^{\prime} \bar{g}^{\prime} \bar{y}\right]} \\
& \quad \times \frac{\left[y, h^{\prime} \bar{g}^{\prime} \bar{y}, y g^{\prime} \bar{y}\right]\left[g^{\prime} \bar{y}, y, h^{\prime} \bar{g}^{\prime} \bar{y}\right]\left[h^{\prime} \bar{g}^{\prime}, g^{\prime} \bar{y}, y\right]}{\left[y, g^{\prime} \bar{y}, y h^{\prime} \bar{g}^{\prime} \bar{y}\right]\left[h^{\prime} \bar{g}^{\prime} \bar{y}, y, g^{\prime} \bar{y}\right]\left[g^{\prime}, h^{\prime} \bar{g}^{\prime} \bar{y}, y\right]} \frac{[g \bar{x}, x, g \bar{x}][g, h \bar{y} \bar{x}, x][x, g \bar{x}, x h \bar{y} \bar{x}]}{[g \bar{x}, x, h \bar{g} \bar{x}][h \bar{y}, g \bar{x}, x][x, h \bar{g} \bar{x}, x g \bar{x}]} .
\end{aligned}
$$

Following eq. (6.2), the GSD on a 3-cylinder then becomes

$$
\begin{aligned}
& G S D_{3-\mathrm{cyl}}=\operatorname{Tr} P_{3-\mathrm{cyl}}^{0}=\sum_{\substack{x, h, g \in K_{1} \\
y, h^{\prime}, g^{\prime} \in K_{2}}} \sum_{k \in G} \frac{\delta_{g^{\prime}, \bar{k} g k} \delta_{h^{\prime}, \bar{k} h k} \delta_{g, x g \bar{x}} \delta_{h, x h \bar{x}} \delta_{k, x k \bar{y}}}{\left|K_{1}\right|\left|K_{2}\right|} \\
& \times \frac{[h \bar{y}, g, k \bar{y}, y]\left[k, h^{\prime} \bar{g}^{\prime}, g^{\prime} \bar{y}, y\right]\left[h \bar{g}, k \bar{y}, y, g^{\prime} \bar{y}\right]\left[g, k, h^{\prime} \bar{g}^{\prime} \bar{y}, y\right]\left[k, g^{\prime} \bar{y}, y, h^{\prime} \bar{g}^{\prime} \bar{y}\right]\left[k \bar{y}, y, h^{\prime} \bar{g}^{\prime} \bar{y}, y g^{\prime} \bar{y}\right]}{\left[h \bar{g}, k, g^{\prime} \bar{y}, y\right][g, h \bar{g}, k \bar{y}, y]\left[k, h^{\prime} \bar{g}^{\prime} \bar{y}, y, g^{\prime} \bar{y}\right]\left[k, g^{\prime}, h^{\prime} \bar{g}^{\prime} \bar{y}, y\right]\left[g, k \bar{y}, y, h^{\prime} \bar{g}^{\prime} \bar{y}\right]\left[k \bar{y}, y, g^{\prime} \bar{y}, y h^{\prime} \bar{g}^{\prime} \bar{y}\right]} \\
& \times \frac{[h \bar{g} \bar{x}, x, g \bar{x}, x k \bar{y}][g, h \bar{g} \bar{x}, x, k \bar{y}]\left[x, h \bar{g} \bar{x}, x k \bar{y}, y g^{\prime} \bar{y}\right]\left[g \bar{x}, x, k \bar{y}, y h^{\prime} \bar{g}^{\prime} \bar{y}\right]\left[x, k \bar{y}, y g^{\prime} \bar{y}, y h^{\prime} \bar{g}^{\prime} \bar{y}\right][x, g \bar{x}, x h \bar{g} \bar{x}, x k \bar{y}]}{[h \bar{g}, g \bar{x}, x, k \bar{y}]\left[h \bar{g} \bar{x}, x, k \bar{y}, y g^{\prime} \bar{y}\right][x, h \bar{g} \bar{x}, x g \bar{x}, x k \bar{y}][g \bar{x}, x, h \bar{g} \bar{x}, x k \bar{y}]\left[x, k \bar{y}, y h^{\prime} \bar{g}^{\prime} \bar{y}, y g^{\prime} \bar{y}\right]\left[x, g \bar{x}, x k \bar{y}, y h^{\prime} g^{\prime} \bar{y}\right]} \\
& \times \frac{\left[y, h^{\prime} \bar{g}^{\prime} \bar{y}, y g^{\prime} \bar{y}\right]\left[g^{\prime} \bar{y}, y, h^{\prime} \bar{g}^{\prime} \bar{y}\right]\left[h^{\prime} \bar{g}^{\prime}, g^{\prime} \bar{y}, y\right]}{\left[y, g^{\prime} \bar{y}, y h^{\prime} \bar{g}^{\prime} \bar{y}\right]\left[h^{\prime} \bar{g}^{\prime} \bar{y}, y, g^{\prime} \bar{y}\right]\left[g^{\prime}, h^{\prime} \bar{g}^{\prime} \bar{y}, y\right]} \frac{[h \bar{g} \bar{x}, x, g \bar{x}][g, h \bar{g} \bar{x}, x][x, g \bar{x}, x h \bar{g} \bar{x}]}{[g \bar{x}, x, h \bar{g} \bar{x}][h \bar{g}, g \bar{x}, x][x, h \bar{g} \bar{x}, x g \bar{x}]} .
\end{aligned}
$$

Using delta functions $\delta_{g^{\prime}, \bar{k} g k}, \delta_{h^{\prime}, \bar{k} h k}, \delta_{g, x g \bar{x}}, \delta_{h, x h \bar{x}}, \delta_{k, x k \bar{y}}$, The $G S D_{3 \text {-cyl }}$ becomes

$$
\begin{aligned}
& G S D_{3-\mathrm{cyl}}=\sum_{\substack{x, h, g \in K_{1} \\
y, h^{\prime}, g^{\prime} \in K_{2}}} \sum_{k \in G} \frac{\delta_{g^{\prime}, \bar{k} g k} \delta_{h^{\prime}, \bar{k} h k} \delta_{g, x g \bar{x}} \delta_{h, x h \bar{x}} \delta_{k, x k \bar{y}}}{\left|K_{1}\right|\left|K_{2}\right|} \\
& \times \frac{[h \bar{g}, g, k \bar{y}, y][k, \bar{k} h \bar{g} k, \bar{k} g k \bar{y}, y][h \bar{g}, k \bar{y}, y, \bar{k} g k \bar{y}][g, k, \bar{k} h \bar{g} k \bar{y}, y][k, \bar{k} g k \bar{y}, y, \bar{k} h \bar{g} k \bar{y}][k \bar{y}, y, \bar{k} h \bar{g} k \bar{y}, \bar{k} g k]}{[h \bar{g}, k, \bar{k} g k \bar{y}, y][g, h \bar{g}, k \bar{y}, y][k, \bar{k} h \bar{g} k \bar{y}, y, \bar{k} g k \bar{y}][k, \bar{k} g k, \bar{k} h \bar{g} k \bar{y}, y][g, k \bar{y}, y, \bar{k} h \bar{g} k \bar{y}][k \bar{y}, y, \bar{k} g k \bar{y}, \bar{k} h \bar{g} k]} \\
& \times \frac{[h \bar{y}}{[h \bar{g}, k, \bar{k} g k \bar{y}, y][g, h \bar{g}, k \bar{y}, y][k, \bar{k} h \bar{g} k \bar{y}, y, \bar{k} g k \bar{y}][k, \bar{k} g k, \bar{k} h \bar{g} k \bar{y}, y][g, k \bar{y}, y, \bar{k} h \bar{g} k \bar{y}][k \bar{y}, y, \bar{k} g k \bar{y}, \bar{k} h \bar{g} k]} \\
& \times \frac{[h \bar{g} \bar{x}, x, g \bar{x}, k \overline{]}[g, h \bar{g} \bar{x}, x, k \bar{y}][x, h \bar{g} \bar{x}, k, \bar{k} g k][g \bar{x}, x, k \bar{y}, \bar{k} h \bar{g} k][x, k \bar{y}, \bar{k} g k, \bar{k} h \bar{g} k][x, g \bar{x}, h \bar{g}, k]}{[h \bar{g}, g \bar{x}, x, k \bar{y}][h \bar{g} \bar{x}, x, k \bar{y} \bar{k} g k][x, h \bar{g} \bar{x}, g, k][g \bar{x}, x, h \bar{g} \bar{x}, k][x, k \bar{y}, \bar{k} h \bar{g} k, \bar{k} g k][x, g \bar{x}, k, \bar{k} h \bar{g} k]} \\
& \times \frac{[y, \bar{k} h \bar{g} k \bar{y}, \bar{k} g k][\bar{k} g k \bar{y}, \bar{k} h \bar{g} k \bar{y}][\bar{k} h \bar{g} k, \bar{k} g k \bar{y}, y]}{[y, \bar{k} g k \bar{y}, \bar{k} h \bar{g} k][\bar{k} h \bar{g} k \bar{y}, y, \bar{k} g k \bar{y}][\bar{k} g k, \bar{k} h \bar{g} k \bar{y}, y]} \frac{[h \bar{g} \bar{x}, x, g \bar{x}][g, h \bar{g} \bar{x}, x][x, g \bar{x}, h \bar{g}]}{[g \bar{x}, x, h \bar{g} \bar{x}][h \bar{g}, g \bar{x}, x][x, h \bar{g} \bar{x}, g]} .
\end{aligned}
$$


Using the normalization condition (2.3) and the following instances of the generalized Frobenius condition (3.11),

$$
\begin{aligned}
{[a, b, \bar{x}, x] \cdot \mathrm{d}[a, b, \bar{x}, x] } & =1 \\
{[b, \bar{x}, x, a \bar{x}] \cdot \mathrm{d}[b, \bar{x}, x, a \bar{x}] } & =1 \\
{[x, \bar{x}, x a \bar{x}, x b \bar{x}] \cdot \mathrm{d}[x, \bar{x}, x a \bar{x}, x b \bar{x}] } & =1 \\
{[\bar{x}, x, \bar{x}, x a \bar{x}] \cdot \mathrm{d}[x, x, \bar{x}, x a \bar{x}] } & =1,
\end{aligned}
$$

the GSD can be rewritten as $G S D_{3-\mathrm{cyl}}=\sum_{\substack{x, h, g \in K_{1} \\ y, h^{\prime}, g^{\prime} \in K_{2}}} \sum_{k \in G} \frac{\delta_{g^{\prime}, \bar{k} g k} \delta_{h^{\prime}, \bar{k} h k} \delta_{g, x g \bar{x}} \delta_{h, x h \bar{x}} \delta_{k, x k \bar{y}}}{\left|K_{1}\right|\left|K_{2}\right|} F(x, h \bar{g}, g, k)$. $G(y, h \bar{g}, k) \cdot \frac{\eta^{g}(h \bar{g}, x)}{\eta^{k g k}(\bar{k} h \bar{g} k, y)}$, where

$$
\begin{aligned}
F(x, h \bar{g}, g, k)= & \frac{[h \bar{g}, g, \bar{x}, x][h \bar{y}, \bar{x}, x, g \bar{x}][x, \bar{x}, h \bar{g}, g][\bar{x}, x, \bar{x}, g][h \bar{x} \bar{x}, x, g \bar{x}, k][g, h \bar{g} \bar{x}, x, \bar{x} k][x, g \bar{x}, h \bar{g}, k]}{[g, h \bar{y}, \bar{x}, x][g, \bar{x}, x, h \bar{x} \bar{x}][x, \bar{x}, g, h \bar{x}][\bar{x}, x, \bar{x}, h \bar{g}][g \bar{x}, x, h \bar{g} \bar{x}, k][h \bar{y}, g \bar{x}, x, \bar{x} k][x, h \bar{g} \bar{x}, g, k]} \\
& \times \frac{[x, h \bar{g} \bar{x}, k, \bar{k} g k][g \bar{x}, x, \bar{x} k, \bar{k} h \bar{g} k][x, \bar{x} k, \bar{k} g k, \bar{k} h \bar{g} k]}{[x, g \bar{x}, k, \bar{k} h \bar{g} k][h \bar{g} \bar{x}, x, \bar{x} k, \bar{k} g k][x, \bar{x} k, \bar{k} h \bar{g} k, \bar{k} g k]}, \\
G(y, h \bar{g}, g, k)= & \frac{[\bar{k} g k, \bar{k} h \bar{g} k, \bar{y}, y][\bar{k} g k, \bar{y}, y, \bar{k} h \bar{g} k \bar{y}][y, \bar{y}, \bar{k} g k, \bar{k} h \bar{g} k][\bar{y}, y, \bar{y}, \bar{k} h \bar{g} k][k \bar{y}, y, \bar{k} h \bar{g} k \bar{y}, \bar{k} g k]}{[\bar{k} h \bar{g} k, \bar{k} g k, \bar{y}, y][\bar{k} h \bar{g} k, \bar{y}, y, \bar{k} g k \bar{y}][y, \bar{y}, \bar{k} h \bar{g} k, \bar{k} g k][\bar{y}, y, \bar{y}, \bar{k} g k][k \bar{y}, y, \bar{k} g k \bar{y}, \bar{k} h \bar{g} k]} \\
& \times \frac{[k, \bar{k} g k \bar{y}, y, \bar{k} h \bar{g} k \bar{y}][k, \bar{k} h \bar{g} k, \bar{k} g k \bar{y}, y][h \bar{g}, g, k \bar{y}, y][g, k, \bar{k} h \bar{g} k \bar{y}, y][h \bar{g}, k \bar{y}, y, \bar{k} g k \bar{y}]}{[k, \bar{k} h \bar{g} k \bar{y}, y, \bar{k} g k \bar{y}][k, \bar{k} g k, \bar{k} h \bar{g} k \bar{y}, y][g, h \bar{y}, k \bar{y}, y][h \bar{y}, k, \bar{k} g k \bar{y}, y][g, k \bar{y}, y, \bar{k} h \bar{g} k \bar{y}]},
\end{aligned}
$$

If we swap $h \bar{g}$ and $g$ in the definitions above, we find that $F(x, h \bar{g}, g, k) \rightarrow F(x, h \bar{g}, g, k)^{-1}$ and $G(y, h \bar{g}, g, k) \rightarrow G(y, h \bar{g}, g, k)^{-1}$. This implies that $F(x, h \bar{g}, g, k)$ and $G(y, h \bar{g}, g, k)$ have the same mathematical form $[*, *]_{h \bar{g}, g}$.

We first simplify $F(x, h \bar{g}, g, k)$ using the normalization condition (2.3) and the following instances of the 4-cocycle condition (2.4).

$$
\begin{aligned}
\frac{[x, h \bar{g} \bar{x}, k, \bar{k} g k][g \bar{x}, x, \bar{x} k, \bar{k} h \bar{g} k]}{[h \bar{g} \bar{x}, x, \bar{x} k, \bar{k} g k][x, g \bar{x}, k, \bar{k} h \bar{g} k]} & =\frac{[x, h \bar{g}, \bar{x} k, \bar{k} g k][g, x, \bar{x} k, \bar{k} h \bar{g} k][x, h \bar{g} \bar{x}, x, \bar{x} g k][x, g \bar{x}, x, \bar{x} k]}{[h \bar{g}, x, \bar{x} k, \bar{k} g k][x, g, \bar{x} k, \bar{k} h \bar{g} k][x, h \bar{g} \bar{x}, x, \bar{x} k][x, g \bar{x}, x, \bar{x} h \bar{g} k]}, \\
\frac{[h \bar{g} \bar{x}, x, g \bar{x}, k][x, h \bar{g} \bar{x}, x, \bar{x} g k]}{[x, h \bar{g} \bar{x}, g, k]} & =\frac{[h \bar{g}, x, g \bar{x}, k][x, h \bar{g} \bar{x}, x, g \bar{x}]}{[x, h \bar{g}, g \bar{x}, k]}, \\
\frac{[x, g \bar{x}, h \bar{g}, k]}{[g \bar{x}, x, h \bar{g} \bar{x}, k][x, g \bar{x}, x, \bar{x} h \bar{g} k]} & =\frac{[x, g, h \bar{g} \bar{x}, k]}{[g, x, h \bar{g} \bar{x}, k][x, g \bar{x}, x, h \bar{g} \bar{x}]}, \\
\frac{[x, g, h \bar{g} \bar{x}, k]}{[x, h \bar{g}, g \bar{x}, k]} & =\frac{[g, h \bar{g}, \bar{x}, k][x, g, h \bar{g}, \bar{x} k][x h \bar{g}, g, \bar{x}, k][x, h \bar{g}, g, \bar{x} k]}{[x g, h \bar{g}, \bar{x}, k][x, g, h \bar{g}, \bar{x}][h \bar{g}, g, \bar{x}, k][x, h \bar{g}, g, \bar{x} k]} \\
\frac{[h \bar{g}, x, g \bar{x}, k][x, g \bar{x}, x, \bar{x} k]}{[g, x, h \bar{x} \bar{x}, k][x, h \bar{g} \bar{x}, x, \bar{x} k]} & =\frac{[h \bar{g}, x, g, \bar{x} k][g, h \bar{g}, x, \bar{x} k][h \bar{g}, x, g \bar{x}, x][h \bar{g} \bar{x}, g \bar{x}, x, \bar{x} k]}{[h \bar{g}, g, x, \bar{x} k][g, x, h \bar{g}, \bar{x} k][g, x, h \bar{g} \bar{x}, x][g x, h \bar{g} \bar{x}, x, \bar{x} k]} .
\end{aligned}
$$

Note that

$$
[x, \bar{x} k]_{g, h \bar{g}}=\frac{[g, h \bar{g}, x, \bar{x} k][g, x, \bar{x} k, \bar{k} h \bar{g} k][x, h \bar{g}, \bar{x} k, \bar{k} g k][x, \bar{x} k, \bar{k} g k, \bar{k} h \bar{g} k][h \bar{g}, x, g, \bar{x} k][x, g, h \bar{g}, \bar{x} k]}{[h \bar{g}, g, x, \bar{x} k][g, x, h \bar{g}, \bar{x} k][h \bar{g}, x, \bar{x} k, \bar{k} g k][x, \bar{x} k, \bar{k} h \bar{g} k, \bar{k} g \bar{k}][x, h \bar{g}, g, \bar{x} k][x, g, \bar{x} k, \bar{k} h \bar{g} k]} .
$$

We then obtain

$$
\begin{aligned}
F(x, h \bar{g}, g, k)=[x, \bar{x} k]_{g, h \bar{g}} \times \frac{[g, h \bar{g} \bar{x}, x, \bar{x} k][h \bar{g}, g, \bar{x}, x][h \bar{g}, \bar{x}, x, g \bar{x}][x, \bar{x}, h \bar{g}, g][\bar{x}, x \bar{x}, x, g][g, h \bar{g}, \bar{x}, k]}{[h \bar{g}, g \bar{x}, x, \bar{x} k][g, h \bar{g}, \bar{x}, x][g, \bar{x}, x, h \bar{g} \bar{x}][x, \bar{x}, g, h \bar{g}][\bar{x}, x, \bar{x}, h \bar{g}][h \bar{g}, g, \bar{x}, k]} \\
\times \frac{[x h \bar{g}, g, \bar{x}, k][x, h \bar{g}, g, \bar{x}][x, h \bar{g} \bar{x}, x, g \bar{x}][h \bar{g}, x, g \bar{x}, x][x h \bar{g}, g \bar{x}, x, \bar{x} k]}{[x g, h \bar{g}, \bar{x}, k][x, g, h \bar{g}, \bar{x}][x, g \bar{x}, x, h \bar{g} x][g, x, h \bar{g} \bar{x}, x][x g, h \bar{g} \bar{x}, x, \bar{x} k]}
\end{aligned}
$$


Keep using relations derived from the 4-cocylce condition (2.4), we can simplify $F(x, h \bar{g}, g, k)$ further. Namely, by

$$
\begin{aligned}
\frac{[h \bar{g}, g, \bar{x}, x][g, h \bar{g}, \bar{x}, k][g, h \bar{g} \bar{x}, x, \bar{x} k]}{[g, h \bar{g}, \bar{x}, x][h \bar{g}, g, \bar{x}, k][h \bar{g}, g \bar{x}, x, \bar{x} k]} & =\frac{[g, \bar{x}, x, \bar{x} k]}{[h \bar{g}, \bar{x}, x, \bar{x} k]}, \\
\frac{[x h \bar{g}, g, \bar{x}, k][x h \bar{g}, g \bar{x}, x, \bar{x} k][g, \bar{x}, x, \bar{x} k]}{[x g, h \bar{g}, \bar{x}, k][x g, h \bar{g} \bar{x}, x, \bar{x} k][h \bar{g}, \bar{x}, x, \bar{x} k]} & =\frac{[x h \bar{g}, g, \bar{x}, x]}{[x g, h \bar{g}, \bar{x}, x]}, \\
\frac{[x, h \bar{g}, g, \bar{x}][x h \bar{g}, g, \bar{x}, x]}{[x, g, h \bar{g}, \bar{x}][x g, h \bar{g}, \bar{x}, x]} & =\frac{[h \bar{g}, g, \bar{x}, x][x, g, h \bar{g} \bar{x}, x]}{[g, h \bar{g}, \bar{x}, x][x, h \bar{g}, g \bar{x}, x]}, \\
\frac{[h \bar{g}, \bar{x}, x, g \bar{x}][\bar{x}, x, \bar{x}, g]}{[g, \bar{x}, x, h \bar{g} \bar{x}][\bar{x}, x, \bar{x}, h \bar{g}]} & =\frac{[h \bar{x} \bar{x}, x, \bar{x}, g][h \bar{g}, \bar{x}, x, \bar{x}]}{[g \bar{x}, x, \bar{x}, h \bar{g}][g, \bar{x}, x, \bar{x}]}, \\
\frac{[h \bar{g} \bar{x}, x, \bar{x}, g][x, h \bar{g} \bar{x}, x, g \bar{x}]}{[g \bar{x}, x, \bar{x}, h \bar{g}][x, g \bar{x}, x, h \bar{g} \bar{x}]} & =\frac{[h \bar{g}, x, \bar{x}, g][x, g, \bar{x}, h \bar{g}][x, h \bar{g} \bar{x}, x, \bar{x}]}{[g, x, \bar{x}, h \bar{g}][x, h \bar{g}, \bar{x}, g][x, g \bar{x}, x, \bar{x}]}, \\
\frac{[h \bar{g}, x, g \bar{x}, x][x, g, h \bar{g} \bar{x}, x]}{[g, x, h \bar{g} \bar{x}, x][x, h \bar{g}, g \bar{x}, x]} & =\frac{[x, g, \bar{x}, x][h \bar{g}, x g, \bar{x}, x][g, x, h \bar{g}, \bar{x}][g, h \bar{g}, \bar{x}, x][x, h \bar{g}, g, \bar{x}]}{[x, h \bar{g}, \bar{x}, x][g, x h \bar{g}, \bar{x}, x][h \bar{g}, x, g, \bar{x}][h \bar{g}, g, \bar{x}, x][x, g, h \bar{g}, \bar{x}]}, \\
\frac{[h \bar{g}, x g, \bar{x}, x]}{[g, x h \bar{g}, \bar{x}, x]} & =\frac{[h \bar{g}, g, x, \bar{x}][h \bar{g}, x, \bar{x}, x]}{[g, h \bar{g}, x, \bar{x}][g, x, \bar{x}, x]}, \\
\frac{[x, g, \bar{x}, x][x, h \bar{g} \bar{x}, x, \bar{x}][h \bar{g}, x, \bar{x}, x]}{[x, h \bar{g}, \bar{x}, x][x, g \bar{x}, x, \bar{x}][g, x, \bar{x}, x]} & =\frac{[h \bar{g} \bar{x}, x, \bar{x}, x]}{[g \bar{x}, x, \bar{x}, x]}, \\
\frac{[h \bar{g}, \bar{x}, x, \bar{x}][h \bar{g} \bar{x}, x, \bar{x}, x]}{[g, \bar{x}, x, \bar{x}][g \bar{x}, x, \bar{x}, x]} & =1,
\end{aligned}
$$

we obtain

$$
F(x, h \bar{g}, g, k)=\eta^{g}(h \bar{g}, x) \times \frac{[x, \bar{x} k]_{g, h \bar{g}}}{[x, \bar{x}]_{g, h \bar{g}}}=\eta^{g}(h \bar{g}, x)[\bar{x}, k]_{h \bar{g}, g}
$$

where doubly-twisted cocycle condition has been used

$$
\left.\frac{[y, z]_{\bar{x} w x, \bar{x} u x}[x, y z]_{w, u}}{[x y, z]_{w, u}[x, y]_{w, u}}\right|_{w u=u w}=1 .
$$

Similarly, we can simplify $G(y, h \bar{g}, g, k)$. Using the relations

$\frac{[h \bar{g}, k \bar{y}, y, \bar{k} g k \bar{y}][g, k, \bar{k} h \bar{g} k \bar{y}, y]}{[h \bar{g}, k, \bar{k} g k \bar{y}, y][g, k \bar{y}, y, \bar{k} h \bar{g} k \bar{y}]}=\frac{[h \bar{g}, k \bar{y}, y, \bar{k} g k][g, k \bar{y}, \bar{k} h \bar{g} k, y][k \bar{y}, y, \bar{k} g k \bar{y}, y][g k \bar{y}, y, \bar{k} h \bar{g} k \bar{y}, y]}{[h \bar{g}, k \bar{y}, \bar{k} g k, y][g, k \bar{y}, y, \bar{k} h \bar{g} k][h \bar{g} k \bar{y}, y, \bar{k} g k \bar{y}, y][k \bar{y}, y, \bar{k} h \bar{g} k \bar{y}, y]}$, $\frac{[k, \bar{k} g k \bar{y}, y, \bar{k} h \bar{g} k \bar{y}][g k \bar{y}, y, \bar{k} h \bar{g} k \bar{y}, y]}{[k, \bar{k} g k, \bar{k} h \bar{g} k \bar{y}, y]}=\frac{[\bar{k} g k \bar{y}, y, \bar{k} h \bar{g} k \bar{y}, y][k, \bar{k} g k \bar{y}, y, \bar{k} h \bar{g} k]}{[k, \bar{k} g k \bar{y}, \bar{k} h \bar{g} k, y]}$,

$$
\begin{aligned}
& \begin{array}{l}
{[k, \bar{k} h \bar{g} k, \bar{k} g k \bar{y}, y]} \\
{[k, \bar{k} h \bar{g} k \bar{y}, \bar{k} g k, y]}
\end{array} \\
& \overline{[k, \bar{k} h \bar{g} k \bar{y}, y, \bar{k} g k \bar{y}][h \bar{g} k \bar{y}, y, \bar{k} g k \bar{y}, y]}=\frac{[\bar{k} h \bar{g} k \bar{y}, y, \bar{k} g k \bar{y}, y][k, \bar{k} h \bar{g} k \bar{y}, y, \bar{k} g k]}{\left[{ }^{\prime}\right.}, \\
& \frac{[k, \bar{k} h \bar{g} k \bar{y}, \bar{k} g k, y]}{[k, \bar{k} g k \bar{y}, \bar{k} h \bar{g} k, y]}=\frac{[k \bar{y}, \bar{k} h \bar{g} k, \bar{k} g k, y][k, \bar{y}, \bar{k} h \bar{g} k, \bar{k} g k][\bar{y}, \bar{k} g k, \bar{k} h \bar{g} k, y][k, \bar{y}, \bar{k} g k, \bar{k} h \bar{g} k y]}{[k \bar{y}, \bar{k} g k, \bar{k} h \bar{g} k, y][k, \bar{y}, \bar{k} g k, \bar{k} h \bar{g} k][\bar{y}, \bar{k} h \bar{g} k, \bar{k} g k, y][k, \bar{y}, \bar{k} h \bar{g} k, \bar{k} g k y]}, \\
& \frac{[k \bar{y}, y, \bar{k} g k \bar{y}, y][k, \bar{k} g k \bar{y}, y, \bar{k} h \bar{g} k]}{[k \bar{y}, y, \bar{k} h \bar{g} k \bar{y}, y][k, \bar{k} h \bar{g} k \bar{y}, y, \bar{k} g k]}=\frac{[y, \bar{k} g k \bar{y}, y, \bar{k} h \bar{g} k][k \bar{y}, \bar{k} g k, y, \bar{k} h \bar{g} k][k \bar{y}, y, \bar{k} g k \bar{y}, \bar{k} h \bar{g} k y][k \bar{y}, y, \bar{k} h \bar{g} k, \bar{k} g k]}{[y, \bar{k} h \bar{g} k \bar{y}, y, \bar{k} g k][k \bar{y}, \bar{k} h \bar{g} k, y, \bar{k} g k][k \bar{y}, y, \bar{k} h \bar{g} k \bar{y}, \bar{k} g k y][k \bar{y}, y, \bar{k} g k, \bar{k} h \bar{g} k]},
\end{aligned}
$$

we have

$$
\begin{array}{r}
G(y, h \bar{g}, g, k)=[k \bar{y}, y]_{h \bar{y}, g} \frac{[\bar{k} g k, \bar{k} h \bar{g} k, \bar{y}, y][\bar{k} g k, \bar{y}, y, \bar{k} h \bar{g} k \bar{y}][y, \bar{y}, \bar{k} g k, \bar{k} h \bar{g} k][\bar{y}, y, \bar{y}, \bar{k} h \bar{g} k][k \bar{y}, y, \bar{k} h \bar{g} k \bar{y}, \bar{k} g k]}{[\bar{k} h \bar{g} k, \bar{k} g k, \bar{y}, y][\bar{k} h \bar{g} k, \bar{y}, y, \bar{k} g k \bar{y}][y, \bar{y}, \bar{k} h \bar{g} k, \bar{k} g k][\bar{y}, y, \bar{y}, \bar{k} g k][k \bar{y}, y, \bar{k} g k \bar{y}, \bar{k} h \bar{g} k]} \\
\times \frac{[\bar{k} g k \bar{y}, y, \bar{k} h \bar{g} k \bar{y}, y][k, \bar{y}, \bar{k} h \bar{g} k, \bar{k} g k][\bar{y}, \bar{k} g k, \bar{k} h \bar{g} k, y][k, \bar{y}, \bar{k} g k, \bar{k} h \bar{g} k y][y, \bar{k} g k \bar{y}, y, \bar{k} h \bar{g} k][k \bar{y}, y, \bar{k} g k \bar{y}, \bar{k} h \bar{g} k y]}{\left[\bar{k}_{\bar{g}} k \bar{y}, y, \bar{k} g k \bar{y}, y\right][k, \bar{y}, \bar{k} g k, \bar{k} h \bar{g} k][\bar{y}, \bar{k} h \bar{g} k, \bar{k} g k, y][k, \bar{y}, \bar{k} h \bar{g} k, \bar{k} g k y][y, \bar{k} h \bar{g} k \bar{y}, y, \bar{k} g k][k \bar{y}, y, \bar{k} h \bar{g} k \bar{y}, \bar{k} g k y]} .
\end{array}
$$


Rewriting the above again by the following relations

$$
\begin{aligned}
& \frac{[y, \bar{y}, \bar{k} g k, \bar{k} h \bar{g} k][k \bar{y}, y, \bar{k} h \bar{g} k \bar{y}, \bar{k} g k][k, \bar{y}, \bar{k} h \bar{g} k, \bar{k} g k]}{[y, \bar{y}, \bar{k} h \bar{g} k, \bar{k} g k][k \bar{y}, y, \bar{k} g k \bar{y}, \bar{k} h \bar{g} k][k, \bar{y}, \bar{k} g k, \bar{k} h \bar{g} k]}=\frac{[k \bar{y}, y, \bar{y}, \bar{k} g k]}{[k \bar{y}, y, \bar{y}, \bar{k} h \bar{g} k]}, \\
& \frac{[k, \bar{y}, \bar{k} g k, \bar{k} h \bar{g} k y][k \bar{y}, y, \bar{k} g k \bar{y}, \bar{k} h \bar{g} k y][k \bar{y}, y, \bar{y}, \bar{k} g k]}{[k, \bar{y}, \bar{k} h \bar{g} k, \bar{k} g k y][k \bar{y}, y, \bar{k} h \bar{g} k \bar{y}, \bar{k} g k y][k \bar{y}, y, \bar{y}, \bar{k} h \bar{g} k]}=\frac{[y, \bar{y}, \bar{k} g k, \bar{k} h \bar{g} k y]}{[y, \bar{y}, \bar{k} h \bar{g} k, \bar{k} g k y]}, \\
& \frac{[\bar{y}, \bar{k} g k, \bar{k} h \bar{g} k, y][y, \bar{y}, \bar{k} g k, \bar{k} h \bar{g} k y]}{[\bar{y}, \bar{k} h \bar{g} k, \bar{k} g k, y][y, \bar{y}, \bar{k} h h k, \bar{k} g k y]}=\frac{[y, \bar{y}, \bar{k} g k, \bar{k} h \bar{g} k][y, \bar{k} h \bar{g} k \bar{y}, \bar{k} g k, y]}{[y, \bar{y}, \bar{k} h \bar{g} k, \bar{k} g k][y, \bar{k} g k \bar{y}, \bar{k} h \bar{g} k, y]}, \\
& \frac{[\bar{k} g k, \bar{y}, y, \bar{k} h \bar{g} k \bar{y}][\bar{k} g k \bar{y}, y, \bar{k} h \bar{g} k \bar{y}, y]}{[\bar{k} h \bar{g} k, \bar{y}, y, \bar{k} g k \bar{y}][\bar{k} h \bar{g} k \bar{y}, y, \bar{k} g k \bar{y}, y]}=\frac{[\bar{y}, y, \bar{k} h \bar{g} k \bar{y}, y][\bar{k} h \bar{g} k, \bar{y}, \bar{k} g k, y][\bar{k} g k, \bar{y}, y, \bar{k} h \bar{g} k]}{[\bar{y}, y, \bar{k} g k \bar{y}, y][\bar{k} g k, \bar{y}, \bar{k} h \bar{g} k, y][\bar{k} h \bar{g} k, \bar{y}, y, \bar{k} g k]}, \\
& \frac{[y, \bar{k} g k \bar{y}, y, \bar{k} h \bar{g} k][y, \bar{k} h \bar{g} k \bar{y}, \bar{k} g k, y]}{[y, \bar{k} h \bar{g} k \bar{y}, y, \bar{k} g k][y, \bar{k} g k \bar{y}, \bar{k} h \bar{g} k, y]} \\
&=\frac{[y, \bar{y}, \bar{k} g k y, \bar{k} h \bar{g} k][y, \bar{y}, \bar{k} g k, y][\bar{y}, \bar{k} h \bar{g} k, y, \bar{k} g k][\bar{y}, \bar{k} g k, \bar{k} h \bar{g} k, y][y, \bar{y}, \bar{k} h \bar{g} k, \bar{k} g k]}{[y, \bar{y}, \bar{k} h \bar{g} k y, \bar{k} g k][y, \bar{y}, \bar{k} h \bar{g} k, y][\bar{y}, \bar{k} g k, y, \bar{k} h \bar{g} k][\bar{y}, \bar{k} h \bar{g} k, \bar{k} g k, y][y, \bar{y}, \bar{k} g k, \bar{k} h \bar{g} k]}, \\
& \frac{[y, \bar{y}, \bar{k} g k y, \bar{k} h \bar{g} k]}{[y, \bar{y}, \bar{k} h \bar{g} k y, \bar{k} g k]}=\frac{[y, \bar{y}, y, \bar{k} h \bar{g} k][\bar{y}, y, \bar{k} g k, \bar{k} h \bar{g} k]}{[y, \bar{y}, y, \bar{k} g k][\bar{y}, y, \bar{k} h \bar{g} k, \bar{k} g k]}, \\
& \frac{[\bar{y}, y, \bar{k} h \bar{g} k \bar{y}, y][\bar{y}, y, \bar{y}, \bar{k} h \bar{g} k][y, \bar{y}, \bar{k} g k, y]}{[\bar{y}, y, \bar{k} g k \bar{y}, y][\bar{y}, y, \bar{y}, \bar{k} g k][y, \bar{y}, \bar{k} h \bar{g} k, y]}=\frac{[\bar{y}, y, \bar{y}, \bar{k} h \bar{g} k y]}{[\bar{y}, y, \bar{y}, \bar{k} g k y]}, \\
& \frac{[\bar{y}, y, \bar{y}, \bar{k} h \bar{y} k y][y, \bar{y}, y, \bar{k} h \bar{g} k]}{[\bar{y}, y, \bar{y}, \bar{k} g k y][y, \bar{y}, y, \bar{k} g k]}=1,
\end{aligned}
$$

we arrive at the result

$$
G(y, h \bar{g}, g, k)=\frac{[k \bar{y}, y]_{h \bar{g}, g}}{[\bar{y}, y]_{\bar{k} h \bar{g} k, \bar{k} g k}} \times \frac{1}{\eta^{\bar{k} g k}(\bar{k} h \bar{g} k, y)}=\frac{1}{[k, \bar{y}]_{h \bar{g}, g}} \times \frac{1}{\eta^{\bar{k} g k}(\bar{k} h \bar{g} k, y)} .
$$

These establish the GSD formula eq. (6.3).

Open Access. This article is distributed under the terms of the Creative Commons Attribution License (CC-BY 4.0), which permits any use, distribution and reproduction in any medium, provided the original author(s) and source are credited.

\section{References}

[1] X.G. Wen, Vacuum Degeneracy of Chiral Spin States in Compactified Space, Phys. Rev. B 40 (1989) 7387 [INSPIRE].

[2] X.G. Wen, F. Wilczek and A. Zee, Chiral Spin States and Superconductivity, Phys. Rev. B 39 (1989) 11413 [InSPIRE].

[3] X.G. Wen, Topological Order in Rigid States, Int. J. Mod. Phys. B 4 (1990) 239 [InSPIRE].

[4] X.G. Wen and Q. Niu, Ground-state degeneracy of the fractional quantum Hall states in the presence of a random potential and on high-genus Riemann surfaces, Phys. Rev. B 41 (1990) 9377 [InSPIRE].

[5] A.Yu. Kitaev, Fault tolerant quantum computation by anyons, Annals Phys. 303 (2003) 2 [quant-ph/9707021] [INSPIRE].

[6] M.A. Levin and X.-G. Wen, String-net condensation: A Physical mechanism for topological phases, Phys. Rev. B 71 (2005) 045110 [cond-mat/0404617] [INSPIRE].

[7] A. Kitaev, Anyons in an exactly solved model and beyond, Annals Phys. 321 (2006) 2 [INSPIRE]. 
[8] X. Chen, Z.-C. Gu, Z.-X. Liu and X.-G. Wen, Symmetry-protected topological orders in interacting bosonic systems, Science 338 (2012) 1604.

[9] M. Levin and Z.-C. Gu, Braiding statistics approach to symmetry-protected topological phases, Phys. Rev. B 86 (2012) 115109 [arXiv:1202.3120] [INSPIRE].

[10] L.-Y. Hung and Y. Wan, String-Net Models with $Z_{N}$ Fusion Algebra, Phys. Rev. B 86 (2012) 235132 [arXiv: 1207.6169] [InSPIRE].

[11] Y. Hu, S.D. Stirling and Y.-S. Wu, Ground State Degeneracy in the Levin-Wen Model for Topological Phases, Phys. Rev. B 85 (2012) 075107 [arXiv: 1105.5771] [INSPIRE].

[12] Y. Hu, Y. Wan and Y.-S. Wu, Twisted quantum double model of topological phases in two dimensions, Phys. Rev. B 87 (2013) 125114 [arXiv:1211.3695] [INSPIRE].

[13] A. Mesaros and Y. Ran, Classification of symmetry enriched topological phases with exactly solvable models, Phys. Rev. B 87 (2013) 155115 [arXiv:1212.0835] [INSPIRE].

[14] C.-H. Lin and M. Levin, Generalizations and limitations of string-net models, Phys. Rev. B 89 (2014) 195130 [arXiv: 1402.4081] [INSPIRE].

[15] L. Kong and X.-G. Wen, Braided fusion categories, gravitational anomalies and the mathematical framework for topological orders in any dimensions, arXiv:1405.5858 [INSPIRE].

[16] T. Lan, A Classification of (2+1)D Topological Phases with Symmetries, Ph.D. Thesis (2018) [arXiv: 1801.01210] [INSPIRE].

[17] K. Walker and Z. Wang, (3+1)-TQFTs and Topological Insulators, arXiv:1104.2632 [INSPIRE].

[18] C. Wang and M. Levin, Braiding statistics of loop excitations in three dimensions, Phys. Rev. Lett. 113 (2014) 080403 [arXiv:1403.7437] [INSPIRE].

[19] S. Jiang, A. Mesaros and Y. Ran, Generalized Modular Transformations in (3+1)D Topologically Ordered Phases and Triple Linking Invariant of Loop Braiding, Phys. Rev. X 4 (2014) 031048 [arXiv: 1404.1062] [InSPIRE].

[20] J. Wang and X.-G. Wen, Non-Abelian string and particle braiding in topological order: Modular $\mathrm{SL}(3, \mathbb{Z})$ representation and $(3+1)$-dimensional twisted gauge theory, Phys. Rev. B 91 (2015) 035134 [arXiv: 1404.7854] [INSPIRE].

[21] Y. Wan, J.C. Wang and H. He, Twisted Gauge Theory Model of Topological Phases in Three Dimensions, Phys. Rev. B 92 (2015) 045101 [arXiv:1409.3216] [INSPIRE].

[22] C. Wang and M. Levin, Topological invariants for gauge theories and symmetry-protected topological phases, Phys. Rev. B 91 (2015) 165119 [arXiv:1412.1781] [INSPIRE].

[23] A. Bullivant, M. Calçada, Z. Kádár, P. Martin and J.F. Martins, Topological phases from higher gauge symmetry in 3+1 dimensions, Phys. Rev. B 95 (2017) 155118 [arXiv: 1606.06639] [INSPIRE].

[24] A. Bullivant, M. Calcada, Z. Kádár, J.F. Martins and P. Martin, Higher lattices, discrete two-dimensional holonomy and topological phases in $(3+1) D$ with higher gauge symmetry, arXiv: 1702.00868 [INSPIRE].

[25] T. Lan, L. Kong and X.-G. Wen, A classification of 3+1D bosonic topological orders (I): the case when point-like excitations are all bosons, Phys. Rev. X 8 (2018) 021074 [arXiv:1704.04221]. 
[26] C. Delcamp, Excitation basis for $(3+1) d$ topological phases, JHEP 12 (2017) 128 [arXiv: 1709.04924] [INSPIRE].

[27] C. Delcamp and A. Tiwari, From gauge to higher gauge models of topological phases, arXiv: 1802.10104 [INSPIRE].

[28] T. Lan and X.-G. Wen, A classification of 3+1D bosonic topological orders (II): the case when some point-like excitations are fermions, arXiv:1801.08530 [INSPIRE].

[29] M. Cheng, N. Tantivasadakarn and C. Wang, Loop Braiding Statistics and Interacting Fermionic Symmetry-Protected Topological Phases in Three Dimensions, Phys. Rev. X 8 (2018) 011054.

[30] C. Nayak, S.H. Simon, A. Stern, M. Freedman and S. Das Sarma, Non-Abelian anyons and topological quantum computation, Rev. Mod. Phys. 80 (2008) 1083 [InSPIRE].

[31] V. Kalmeyer and R.B. Laughlin, Equivalence of the resonating valence bond and fractional quantum Hall states, Phys. Rev. Lett. 59 (1987) 2095 [INSPIRE].

[32] N. Read and S. Sachdev, Large-N expansion for frustrated quantum antiferromagnets, Phys. Rev. Lett. 66 (1991) 1773 [INSPIRE].

[33] X.-G. Wen, Topological orders and Chern-Simons theory in strongly correlated quantum liquid, Int. J. Mod. Phys. B 5 (1991) 1641 [InSPIRE].

[34] R. Moessner and S.L. Sondhi, Resonating Valence Bond Phase in the Triangular Lattice Quantum Dimer Model, Phys. Rev. Lett. 86 (2001) 1881 [INSPIRE].

[35] K. von Klitzing, G. Dorda and M. Pepper, New method for high accuracy determination of the fine structure constant based on quantized Hall resistance, Phys. Rev. Lett. 45 (1980) 494 [INSPIRE].

[36] D.C. Tsui, H.L. Stormer and A.C. Gossard, Two-dimensional magnetotransport in the extreme quantum limit, Phys. Rev. Lett. 48 (1982) 1559 [InSPIRE].

[37] R.B. Laughlin, Anomalous quantum Hall effect: An Incompressible quantum fluid with fractionallycharged excitations, Phys. Rev. Lett. 50 (1983) 1395 [INSPIRE].

[38] R. Tao and Y.-S. Wu, Gauge invariance and fractional quantum Hall effect, Phys. Rev. B 30 (1984) 1097 [INSPIRE].

[39] G.W. Moore and N. Read, Nonabelions in the fractional quantum Hall effect, Nucl. Phys. B 360 (1991) 362 [InSPIRE].

[40] X.G. Wen, NonAbelian statistics in the fractional quantum Hall states, Phys. Rev. Lett. 66 (1991) 802 [INSPIRE].

[41] R. Willett, J.P. Eisenstein, H.L. Stormer, D.C. Tsui, A.C. Gossard and J.H. English, Observation of an even-denominator quantum number in the fractional quantum Hall effect, Phys. Rev. Lett. 59 (1987) 1776 [INSPIRE].

[42] I.P. Radu, J.B. Miller, C.M. Marcus, M.A. Kastner, L.N. Pfeiffer and K.W. West, Quasi-Particle Properties from Tunneling in the Formula Fractional Quantum Hall State, Science 320 (2008) 899 [INSPIRE].

[43] B. Dittrich, (3+1)-dimensional topological phases and self-dual quantum geometries encoded on Heegaard surfaces, JHEP 05 (2017) 123 [arXiv:1701.02037] [INSPIRE].

[44] C. Delcamp and B. Dittrich, Towards a dual spin network basis for $(3+1)$ d lattice gauge theories and topological phases, arXiv:1806.00456 [INSPIRE]. 
[45] A. Kitaev and L. Kong, Models for Gapped Boundaries and Domain Walls, Commun. Math. Phys. 313 (2012) 351.

[46] M. Levin, Protected edge modes without symmetry, Phys. Rev. X 3 (2013) 021009 [arXiv:1301.7355] [INSPIRE].

[47] L. Kong, Anyon condensation and tensor categories, Nucl. Phys. B 886 (2014) 436 [arXiv: 1307.8244$]$ [INSPIRE].

[48] L.-Y. Hung and Y. Wan, K matrix Construction of Symmetry-Enriched Phases of Matter, Phys. Rev. B 87 (2013) 195103 [arXiv: 1302.2951] [InSPIRE].

[49] L.-Y. Hung and Y. Wan, Ground State Degeneracy of Topological Phases on Open Surfaces, Phys. Rev. Lett. 114 (2015) 076401 [arXiv:1408.0014] [INSPIRE].

[50] L. Kong, X.-G. Wen and H. Zheng, Boundary-bulk relation in topological orders, Nucl. Phys. B 922 (2017) 62 [arXiv:1702.00673] [INSPIRE].

[51] J. Wang and X.-G. Wen, Boundary Degeneracy of Topological Order, Phys. Rev. B 91 (2015) 125124 [arXiv:1212.4863] [INSPIRE].

[52] T. Lan, J.C. Wang and X.-G. Wen, Gapped Domain Walls, Gapped Boundaries and Topological Degeneracy, Phys. Rev. Lett. 114 (2015) 076402 [arXiv:1408.6514] [InSPIRE].

[53] A. Bullivant, Y. Hu and Y. Wan, Twisted quantum double model of topological order with boundaries, Phys. Rev. B 96 (2017) 165138 [arXiv:1706.03611] [INSPIRE].

[54] C. Wang, C.-H. Lin and M. Levin, Bulk-Boundary Correspondence for Three-Dimensional Symmetry-Protected Topological Phases, Phys. Rev. X 6 (2016) 021015.

[55] S.X. Cui, Higher Categories and Topological Quantum Field Theories, arXiv:1610.07628 [INSPIRE].

[56] J. Wang, X.-G. Wen and E. Witten, Symmetric Gapped Interfaces of SPT and SET States: Systematic Constructions, Phys. Rev. X 8 (2018) 031048 [arXiv: 1705. 06728] [INSPIRE].

[57] L. Fidkowski and A. Vishwanath, Realizing anomalous anyonic symmetries at the surfaces of three-dimensional gauge theories, Phys. Rev. B 96 (2017) 045131 [arXiv:1511.01502] [INSPIRE].

[58] D.J. Williamson and Z. Wang, Hamiltonian models for topological phases of matter in three spatial dimensions, Annals Phys. 377 (2017) 311 [arXiv: 1606.07144] [INSPIRE].

[59] Y. Hu, Y. Wan and Y.-S. Wu, Boundary Hamiltonian theory for gapped topological orders, Chin. Phys. Lett. 34 (2017) 077103 [arXiv:1706.00650] [INSPIRE].

[60] Y. Hu, Z.-X. Luo, R. Pankovich, Y. Wan and Y.-S. Wu, Boundary Hamiltonian theory for gapped topological phases on an open surface, JHEP 01 (2018) 134 [arXiv:1706.03329] [INSPIRE].

[61] U. Pachner, Bistellare Äquivalenz kombinatorischer Mannigfaltigkeiten, Arch. Math. 30 (1978) 89.

[62] U. Pachner, Konstruktionsmethoden und das kominatorische Homoomorphieproblem für Triangulationen semilinearer Mannigfaltigkeiten, Ahb. Math. Sem. Univ. Hamburg $\mathbf{5 7}$ (1987) 69.

[63] Y. Hu, Y. Wan and Y.-S. Wu, From effective Hamiltonian to anomaly inflow in topological orders with boundaries, JHEP 08 (2018) 092 [arXiv:1706.09782] [INSPIRE]. 